Dynamics of Continuous, Discrete and Impulsive Systems, Series B, 12 (2005), no. 5-6, 649-687

http:monotone.uwaterloo.ca/ journal

\title{
Towards a Geometric Theory of Hybrid Systems
}

\author{
Slobodan N. Simić ${ }^{1}$ Karl Henrik Johansson ${ }^{2}$ \\ John Lygeros ${ }^{3}$ and Shankar Sastry ${ }^{4}$ \\ ${ }^{1}$ Department of Mathematics \\ San José State University, San José, CA 95192, USA \\ ${ }^{2}$ Department of Signals, Sensors \& Systems, Royal Institute of Technology, 10044 \\ Stockholm, Sweden \\ ${ }^{3}$ Department of Electrical and Computer Engineering, University of Patras, Rio, Patras, \\ GR 26500, Greece \\ ${ }^{4}$ Department of Electrical Engineering and Computer Sciences \\ University of California, Berkeley, CA 94720, USA
}

\begin{abstract}
We propose a framework for a geometric theory of hybrid systems. Given a deterministic, non-blocking hybrid system, we introduce the notion of its hybrifold with the associated hybrid flow on it. This enables us to study hybrid systems from a global geometric perspective as (generally non-smooth) dynamical systems. This point of view is adopted in studying the Zeno phenomenon. We show that it is due to nonsmoothness of the hybrid flow. We introduce the notion of topological equivalence of hybrid systems and locally classify isolated Zeno states in dimension two.
\end{abstract}

Keywords. Hybrid system; dynamical system; hybrifold; Zeno; topological equivalence. AMS (MOS) subject classification: 37C10, 37C99,34C99, 37N35

\section{Introduction}

Any system which changes in time and involves interactions between continuous processes and discrete automata can be viewed as a hybrid system. The name comes from the hybrid nature of its evolution: intervals of continuoustime change are interleaved with instantaneous, discrete jumps. Hybrid systems are a topic of great interest, primarily in the control and computer science communities. Emerging applications in areas such as air traffic management [37], automotive control [5, 1], real-time software verification [2], transportation systems [39, 25], manufacturing [32], mobile robotics [6], and process industry [13], motivate work on hybrid systems modeling [11, 28, 4, $38,10,20]$, analysis [3, 40, 9, 22, 17, 23], and controller design [29, 8, 10, 27, 7]. Although extensive research efforts have been made in the area of hybrid systems, the understanding of the fundamental dynamical properties of systems 
with interacting continuous-time and discrete-event dynamics $[11,20,16,21$, $42]$ is not satisfactory.

In this paper we present a geometric approach for treatment of a class of hybrid systems which resemble deterministic continuous-time piecewise smooth dynamical systems. Early results in this direction where first reported in [33]. We introduce the notions of the hybrifold and hybrid flow, which enable us to study the hybrid system "in one piece", that is, as a single, piecewise smooth dynamical system. It is well known that even simple smooth dynamical systems can exhibit very complicated behavior which makes their global study using analytical methods very difficult. This is why developing qualitative (i.e., geometric and topological) techniques has been at the center of modern dynamics, ever since Poincaré's seminal work at the end of the $19^{\text {th }}$ and beginning of the $20^{\text {th }}$ century. For a beautiful introduction to this field, the reader is referred to [31]. In this article, we make a first step towards developing such techniques for hybrid systems.

Having established a suitable framework, we focus particularly on the Zeno phenomenon, which does not occur in smooth dynamical systems. We study its underlying geometric causes and classify it in dimension two. This classification is with respect to the notion of topological equivalence borrowed from dynamical systems: two systems are equivalent if they are qualitatively the same.

The advantages of the hybrifold method are the following.

- It enables one to use the standard techniques of the theory of dynamical systems to study hybrid systems (which have a hybrifold).

- It provides a convenient framework for global analysis of hybrid systems, giving insight into global behavior of hybrid executions, which is not possible to obtain by local, domain-by-domain, analysis.

- It introduces a convenient setting for qualitative analysis of hybrid systems; for instance, it simplifies the definitions of topological equivalence and structural stability of hybrid systems.

Furthermore, we distill the key properties of a class of examples into a list of assumptions, and study all hybrid systems which share these properties.

The organization of the paper is the following. In Section 2 we define the model, present some basic examples, and formulate the standing assumptions. In Section 3 we introduce and discuss the basic properties of the hybrifold and hybrid flow. Section 4 is concerned with the geometry of the Zeno phenomenon and Section 5 introduces topological equivalence of hybrid systems and classifies isolated Zeno states in dimension two. Finally, we make a few concluding remarks in Section 6. 


\section{Preliminaries}

\section{Definitions and examples}

Let us first define our model and give some basic examples which will be useful later in the paper. The following definitions are motivated by [20, 26].

Definition 2.1. An n-dimensional hybrid system is a 6-tuple

$$
\mathbf{H}=(Q, E, \mathcal{D}, \mathcal{X}, \mathcal{G}, \mathcal{R}),
$$

where:

- $Q=\{1, \ldots, k\}$ is the finite set of (discrete) states of $\mathbf{H}$, where $k \geq 1$ is an integer;

- $E \subset Q \times Q$ is the collection of edges;

- $\mathcal{D}=\left\{D_{i}: i \in Q\right\}$ is the collection of domains of $\mathbf{H}$, where $D_{i} \subset$ $\{i\} \times \mathbb{R}^{n}$, for all $i \in Q$;

- $\mathcal{X}=\left\{X_{i}: i \in Q\right\}$ is the collection of vector fields such that $X_{i}$ is Lipschitz on $D_{i}$ for all $i \in Q$; we denote the local flow of $X_{i}$ by $\left\{\phi_{t}^{i}\right\}$.

- $\mathcal{G}=\{G(e): e \in E\}$ is the collection of guards, where for each $e=$ $(i, j) \in E, G(e) \subset D_{i}$;

- $\mathcal{R}=\left\{R_{e}: e \in E\right\}$ is the collection of resets, where for each $e=(i, j) \in$ $E, R_{e}$ is a relation between elements of $G(e)$ and elements of $D_{j}$, i.e., $R_{e} \subset G(e) \times D_{j}$.

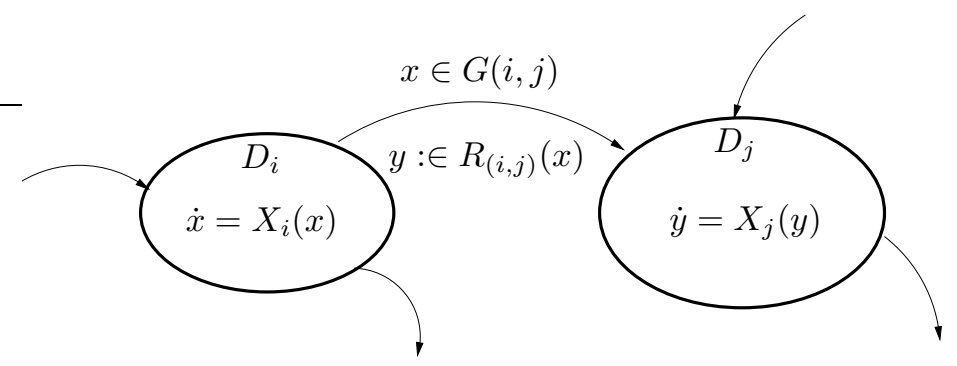

Figure 1: A hybrid system.

Remark 1. (a) Our terminology differs slightly from the usual terminology for hybrid systems, where the domains are called the invariants. Since there is nothing dynamically invariant about these sets, we prefer to reserve the term invariant set for later, more appropriate use. 
(b) We do not consider the set of initial states as a separate item in the definition of a hybrid system. This is because we restrict ourselves to studying only so called non-blocking systems in which all points can be initial conditions. The only assumption we will make subsequently is that the initial state is in one of the domains.

(c) The above definition clearly allows a hybrid system to be a wild object. An important question is: what properties should the domains, guards, resets and vector fields of $\mathbf{H}$ satisfy to get a large enough class of hybrid systems about which something useful can be said? Soon we will deal with this question in detail and focus our attention on one such class of hybrid systems (which we will call regular).

(d) If a reset relation $R_{e}$ is a map $G(e) \rightarrow D_{j}$, with $e=(i, j) \in E$, we write $y=R_{e}(x)$ instead of $(x, y) \in R_{e}$.

(e) We also set

$$
D=\bigcup_{i \in Q} D_{i},
$$

and call this set the total domain of $\mathbf{H}$. If the resets are maps, we write

$$
\begin{gathered}
G=\bigcup_{e \in E} G(e), \quad R=\bigcup_{e \in E} R_{e}(G(e)), \\
\overline{\mathcal{G}}=\{\overline{G(e)}: e \in E\}, \quad \overline{\mathcal{R}}=\left\{\overline{R_{e}(G(e))}: e \in E\right\} .
\end{gathered}
$$

Therefore, $G$ is the union of all guards, $\overline{\mathcal{G}}$ is the collection of closures of all guards, etc.

(f) Observe that domains $D_{i}$ lie in distinct copies of $\mathbb{R}^{n}$, namely $\{i\} \times$ $\mathbb{R}^{n}, i \in Q$. However, to simplify things, we will sometimes abuse the notation and drop the $i$. More precisely, we identify $\{i\} \times \mathbb{R}^{n}$ with $\mathbb{R}^{n}$ via the diffeomorphism $F_{i}:(i, x) \mapsto x$, and write $D_{i}, G(e), R_{e}, X_{i}, \ldots$ for $F_{i}\left(D_{i}\right), F_{i}(G(e)), F_{i} \circ R_{e} \circ F_{j}^{-1}, T F_{i} \circ X_{i} \circ F_{i}^{-1}, \ldots$, where $e=(i, j) \in E$ and $T F_{i}$ is the derivative or tangent map of $F_{i}$.

Given $\mathbf{H}$, the basic idea is that starting from a point in some domain $D_{i}$, we flow according to $X_{i}$ until (and if) we reach some guard $G(i, j)$. At that point it is possible for the state to switch via the reset $R_{(i, j)}$ and continue flowing in $D_{j}$ according to $X_{j}$, and so on.

Example 2.2 (Water Tank $W T$ ). Consider the water tank system discussed in $[4,18,20]$ (Fig. 2). For $i \in Q=\{1,2\}, x_{i}$ denotes the volume of water in tank $i, v_{i}$ is the constant rate of flow of water out of tank $i$, and $l_{i}$ is the desired volume of water in tank $i$. The constant rate of water flow into the system, dedicated exclusively to one tank at a time, is denoted by $w$. The control task is to keep the water volume above $l_{1}$ and $l_{2}$ (assuming the 
initial volumes are above $l_{1}$ and $l_{2}$ respectively) by a strategy that switches the inflow to the first tank whenever $x_{1}=l_{1}$ and to the second tank whenever $x_{2}=l_{2}$.

Here $n=2, k=2, E=\{(1,2),(2,1)\}$,

$$
D_{1}=\{1\} \times C, \quad D_{2}=\{2\} \times C,
$$

where $C=\left[l_{1}, \infty\right) \times\left[l_{2}, \infty\right)$,

$$
X_{1}=\left(w-v_{1},-v_{2}\right)^{T}, \quad X_{2}=\left(-v_{1}, w-v_{2}\right)^{T},
$$

$G(1,2)=\left\{\left(1, x_{1}, x_{2}\right) \in D_{1}: x_{2}=l_{2}\right\}, \quad G(2,1)=\left\{\left(2, x_{1}, x_{2}\right) \in D_{2}: x_{1}=l_{1}\right\}$,

and

$$
R_{(1,2)}\left(1, x_{1}, l_{2}\right)=\left(2, x_{1}, l_{2}\right), \quad R_{(2,1)}\left(2, l_{1}, x_{2}\right)=\left(1, l_{1}, x_{2}\right) .
$$

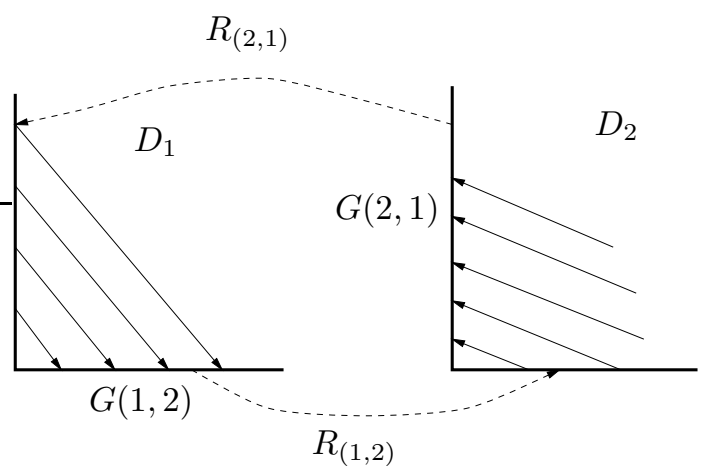

Figure 2: The water tank example.

We will assume that $\max \left(v_{1}, v_{2}\right)<w<v_{1}+v_{2}$. This means that more water is flowing out of than into the tanks.

Example 2.3 (Bouncing Ball $B B$ ). This is a simplified model of an elastic ball that is bouncing and losing a fraction of its energy with each bounce, discussed in [20]. We denote by $x_{1}$ the altitude of the ball and by $x_{2}$ its vertical speed. Here $n=2, k=1, E=\{(1,1)\}$,

$$
\begin{array}{cc}
D_{1}=\left\{\left(1, x_{1}, x_{2}\right): x_{1} \geq 0\right\}, & X_{1}\left(1, x_{1}, x_{2}\right)=\left(x_{2},-g\right)^{T}, \\
G(1,1) & =\left\{\left(1,0, x_{2}\right): x_{2} \leq 0\right\}, \quad R_{(1,1)}\left(1,0, x_{2}\right)=\left(1,0,-c x_{2}\right),
\end{array}
$$

where $g$ is the acceleration due to gravity and $0<c<1$ (Fig. 3).

Example 2.4 (Bouncing $m$-Ball $B B(m)$ ). The only difference between this and the previous example is that we have $m$ different domains in which the ball can bounce. After each bounce, the ball switches to the next domain 


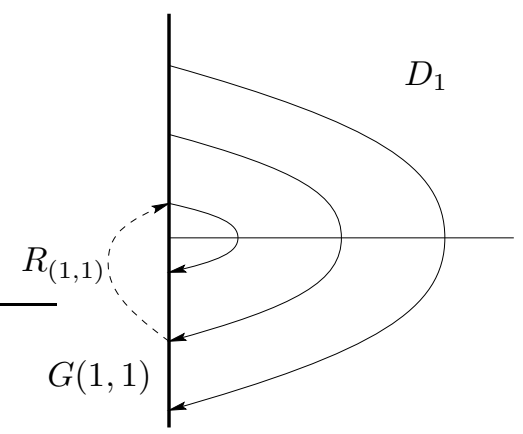

Figure 3: Bouncing ball.

in a cyclical order. That is, $n=2, k=m>1, E=\{(1,2),(2,3), \ldots,(m-$ $1, m),(m, 1)\}$, and for all $i \in Q$,

$$
\begin{gathered}
D_{i}=\{i\} \times\left\{\left(x_{1}, x_{2}\right): x_{1} \geq 0\right\}, G(i, i+1)=\{i\} \times\left\{\left(0, x_{2}\right): x_{2} \leq 0\right\}, \\
R_{(i, i+1)}\left(i, 0, x_{2}\right)=\left(i+1,0,-c x_{2}\right),
\end{gathered}
$$

where we conveniently identify $m+1:=1$. Note that here the domains are just different copies of the closed right half-plane in $\mathbb{R}^{2}$.

Example 2.5 (Ball Bouncing on an $N$-step Staircase $B B S(N)$ ). Here we have a staircase with steps $i=1, \ldots, N$ of width $w_{i}>0$ and height $h_{i}>0$ on which a ball is bouncing. Define $\hat{w}_{m}=\sum_{i=1}^{m} w_{i}$ and $\hat{h}_{m}=\sum_{i=m}^{N} h_{i}$. Assume also that with each bounce the ball loses a proportional amount of its vertical velocity $\left(x_{2}\right)$ and that it has constant horizontal speed $\left(x_{3}\right)$. Denote by $x_{1}$ its vertical position. Then we have: $Q=\{1, \ldots, N+1\}$, $E=\{(i, i): 1 \leq i \leq N+1\} \cup\{(1,2), \ldots,(N, N+1)\}$, and for $1 \leq i \leq N$,

$$
\begin{gathered}
D_{i}=\{i\} \times\left[\hat{h}_{i}, \infty\right) \times(-\infty, \infty) \times\left(\hat{w}_{i-1}, \hat{w}_{i}\right], \\
G(i, i)=\left\{\left(i, x_{1}, x_{2}, x_{3}\right) \in D_{i}: x_{1}=\hat{h}_{i}, x_{2} \leq 0, x_{3}<\hat{w}_{i}\right\}, \\
G(i, i+1)=\left\{\left(i, x_{1}, x_{2}, x_{3}\right) \in D_{i}: x_{3}=\hat{w}_{i}\right\}, \quad R_{(i, i+1)}(i, \mathbf{x})=(i+1, \mathbf{x}) .
\end{gathered}
$$

For $1 \leq i \leq N+1$,

$$
X_{i}\left(i, x_{1}, x_{2}, x_{3}\right)=\left(x_{2},-g, v\right)^{T}, \quad R_{(i, i)}\left(i, x_{1}, x_{2}, x_{3}\right)=\left(i, x_{1},-c x_{2}, x_{3}\right) .
$$

Finally,

$$
\begin{gathered}
G(N+1, N+1)=\left\{\left(N+1, x_{1}, x_{2}, x_{3}\right) \in D_{N+1}: x_{1}=0, x_{2} \leq 0\right\}, \\
D_{N+1}=\{N+1\} \times[0, \infty) \times(-\infty, \infty) \times\left(\hat{w}_{N}, \infty\right) .
\end{gathered}
$$


Example 2.6 (Two Saddles $S 2(\lambda)$ ). Here (see Fig. 4) $n=2, k=2$, $0<\lambda<1, E=\{(1,2),(2,1)\}$, the domains are two copies of the square $S=[-1,1] \times[-1,1]$, i.e. for $i \in Q$,

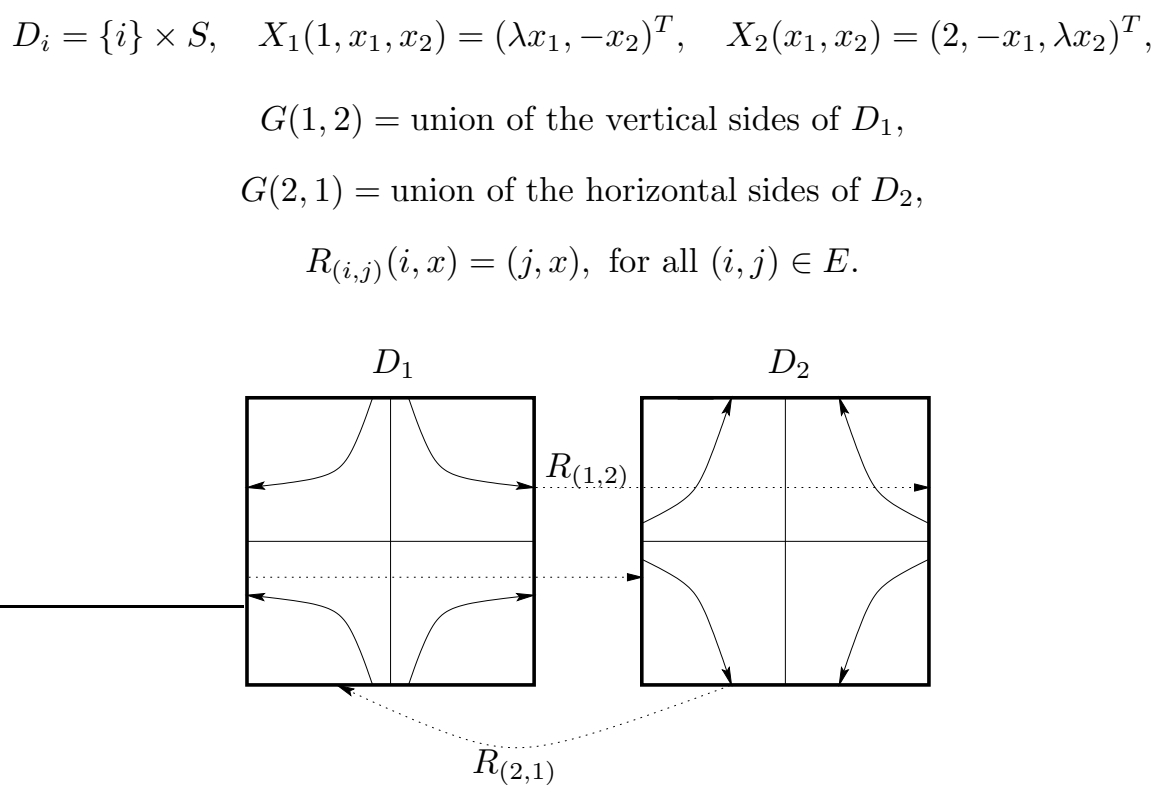

Figure 4: $S 2(\lambda)$

Example 2.7 (Flow on the 2-torus $T^{2}(\alpha)$ ). We have $\alpha>0, n=2, k=2$, $E=\{(1,2),(2,1)\}, D_{i}=\{i\} \times K$, where $K=[0,1] \times[0,1]$ is the unit square, $X_{1}=X_{2}=(1, \alpha)^{T}$ are constant vector fields,

$$
\begin{gathered}
G(i, i)=\{i\} \times S_{\text {upper }}, \quad G(i, j)=\{i\} \times S_{\text {right }}, \\
R_{(i, i)}(i, x, 1)=(i, x, 0) \text { and } R_{(i, j)}(i, 1, y)=(j, 0, y),
\end{gathered}
$$

where $i, j=1,2, i \neq j, S_{\text {upper }}=[0,1] \times\{1\}$ and $S_{\text {right }}=\{1\} \times[0,1)$ denote the (closed) upper and (half-closed) right side of $K$. Note that $R_{(i, i)}(\{i\} \times$ $\left.S_{\text {upper }}\right)=\{i\} \times S_{\text {lower }}$ and $R_{(i, j)}\left(\{i\} \times S_{\text {right }}\right)=\{j\} \times S_{\text {left }}$, with the obvious meaning of $S_{\text {lower }}$ and $S_{\text {left }}$ (see Fig. 5).

If we proceed in the usual way and identify $\{i\} \times S_{\text {upper }}$ with $\{i\} \times S_{\text {lower }}$ via $R_{(i, i)}$ and $\{i\} \times S_{\text {right }}$ with $\{j\} \times S_{\text {left }}$ via $R_{(i, j)}$ (where $i, j=1,2, i \neq j$ ), we obtain the standard 2 -torus with a smooth straight-line flow with slope $\alpha$ on it. This is a "baby-version" of a construction we will later apply to more general hybrid systems.

Keeping in mind the examples above, we now define the notions of a hybrid time trajectory and an execution of a hybrid system. 
Definition 2.8. A (forward) hybrid time trajectory is a sequence (finite or infinite) $\tau=\left\{I_{j}\right\}_{j=0}^{N}$ of intervals such that $I_{j}=\left[\tau_{j}, \tau_{j}^{\prime}\right]$ for all $j \geq 0$ if the sequence is infinite; if $N$ is finite, then $I_{j}=\left[\tau_{j}, \tau_{j}^{\prime}\right]$ for all $0 \leq j \leq N-1$ and $I_{N}$ is either of the form $\left[\tau_{N}, \tau_{N}^{\prime}\right]$ or $\left[\tau_{N}, \tau_{N}^{\prime}\right)$. Furthermore, $\tau_{j} \leq \tau_{j}^{\prime}=\tau_{j+1}$, for all $j$.
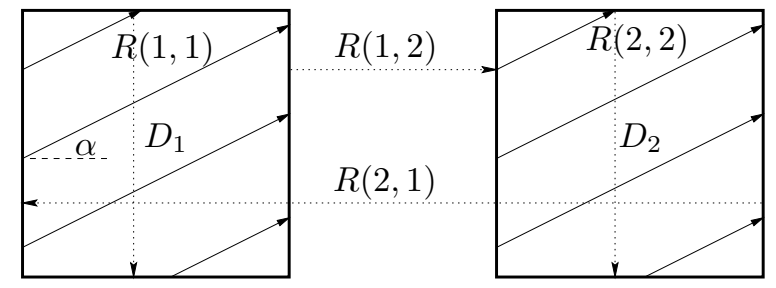

Figure 5: $T^{2}(\alpha)$.

One thinks of $\tau_{j}$ 's as time instants when discrete transitions (or switches) from one domain to another take place. If $\tau=\left\{I_{j}\right\}_{j=0}^{N}$ is a hybrid time trajectory, we will call $N$ its size and denote it by $N(\tau)$. Also, we use $\langle\tau\rangle$ to denote the set $\{0, \ldots, N(\tau)\}$ if $N(\tau)$ is finite, and $\{0,1,2, \ldots\}$ if $N(\tau)$ is infinite.

We will say that $\tau$ is a prefix of a hybrid time trajectory $\tau^{\prime}$ if $N(\tau) \leq$ $N\left(\tau^{\prime}\right)$ (where the inequality is taken in the extended real number system), and for $0 \leq j<N(\tau)$, we have $I_{j}=I_{j}^{\prime}$; furthermore, if $N(\tau)<\infty$, then we must also have $I_{N(\tau)} \subset I_{N\left(\tau^{\prime}\right)}^{\prime}$.

Definition 2.9. An execution (or forward execution) of a hybrid system $\mathbf{H}$ is a triple $\chi=(\tau, q, x)$, where $\tau$ is a hybrid time trajectory, $q:\langle\tau\rangle \rightarrow Q$ is a map, and $x=\left\{x_{j}: j \in\langle\tau\rangle\right\}$ is a collection of $C^{1}$ maps such that $x_{j}: I_{j} \rightarrow D_{q(j)}$ and for all $t \in I_{j}$,

$$
\dot{x}_{j}(t)=X_{q(j)}\left(x_{j}(t)\right) .
$$

Furthermore, for all $j \in\langle\tau\rangle$ such that $j<N(\tau)$, we have

$$
(q(j), q(j+1)) \in E,
$$

and

$$
\left(x_{j}\left(\tau_{j}^{\prime}\right), x_{j+1}\left(\tau_{j+1}\right)\right) \in R_{(q(j), q(j+1))} .
$$

Observe the abuse of notation in (1): the curve $x_{j}(t)$ is in $D_{q(j)} \subset\{q(j)\} \times$ $\mathbb{R}^{n}$, but we identify its tangent with a vector in $\mathbb{R}^{n}$, dropping the discrete part $q(j)$. See also Remark (f) above.

We say that an execution $\chi=(\tau, q, x)$ starts at a point $p \in D$ if $p=x_{0}\left(\tau_{0}\right)$ and $\tau_{0}=0$. It passes through $p$ if $p=x_{j}(t)$ for some $j \in\langle\tau\rangle, t \in I_{j}$. A hybrid system is usually said to accept an execution. Given $p \in D$, it is not difficult 
to see that there are many ways in which a hybrid system can accept several executions starting from or passing through $p$. For instance, this happens if at least one of the resets is a relation which is not a function.

For an execution $\chi=(\tau, q, x)$, denote by $\tau_{\infty}(\chi)$ its (forward) execution time:

$$
\tau_{\infty}(\chi)=\sum_{j=0}^{N(\tau)}\left(\tau_{j}^{\prime}-\tau_{j}\right)=\lim _{j \rightarrow N(\tau)} \tau_{j}^{\prime}-\tau_{0} .
$$

Definition 2.10. An execution $\chi$ is called:

- infinite, if $N(\tau)=\infty$ or $\tau_{\infty}(\chi)=\infty$;

- a Zeno execution if $N(\tau)=\infty$ and $\tau_{\infty}(\chi)<\infty$;

- maximal if it is not a strict prefix of any other execution of $\mathbf{H}$.

In other words, an execution is Zeno if it takes an infinite number of discrete transitions in a finite amount of time. An execution is maximal if there exists no other execution $\chi^{\prime}=\left(\tau^{\prime}, q^{\prime}, x^{\prime}\right)$ such that $\tau$ is a strict prefix of $\tau^{\prime}$ and $x_{j}=x_{j}^{\prime}$ on $I_{j}$ for all $j \in\langle\tau\rangle$.

We will call a Zeno execution static if there exists $l \geq 0$ such that $\tau_{j}^{\prime}=\tau_{j}$, for all $j \geq l$. Otherwise, it will be called dynamic.

In Examples $2.2(W T), 2.3(B B)$, and $2.4(B B(m))$ for every $p \neq(0,0)$, the maximal execution starting from $p$ is dynamic Zeno. The same can be shown for Examples $2.5(B B S(N))$ if $0<c<1$ and $2.6(S 2(\lambda))$ if $0<\lambda<1$ and $p$ belongs to neither axis. On the other hand, every maximal execution in Example $2.7\left(T^{2}(\alpha)\right)$ is infinite with infinite execution time. For more examples and a connection with Filippov solutions in the context of differential equations with discontinuous right-hand sides, see [14].

Definition 2.11. A hybrid system is called deterministic if for every $p \in D$ there exists at most one maximal execution starting from $p$. It is called nonblocking if for every $p \in D$ there is at least one infinite execution starting from $p$.

Necessary and sufficient conditions for a hybrid system to be deterministic and non-blocking can be found in $[26,24,21]$ and for special classes of systems in $[38,19,15]$. Roughly speaking, resets have to be functions, guards have to be mutually disjoint and whenever a continuous trajectory of one of the vector fields in $\mathcal{X}$ is about to exit the domain in which it lies, it has to hit a guard. It is easy to show (using the conditions in $[26,21]$ for example) that all the examples introduced above are non-blocking and deterministic.

\section{Standing assumptions}

To obtain a class of hybrid systems sufficiently tame for our study yet sufficiently broad to include a fair number of important examples, we impose the 
following standing assumptions on the hybrid system $\mathbf{H}=(Q, E, \mathcal{D}, \mathcal{X}, \mathcal{G}, \mathcal{R})$ under consideration. They will hold in the rest of the paper. Observe that our assumptions on the continuous dynamics are very moderate when compared to, for instance, timed hybrid automata; in each domain, we allow general nonlinear vector fields with a reasonable degree of regularity.

The first assumption deals with uniqueness of executions.

(A1) $\mathbf{H}$ is deterministic and non-blocking.

This means that every point in $D$ is the starting point of a unique infinite (and therefore maximal) execution of $\mathbf{H}$.

The second assumption deals with the geometry of domains.

(A2) There exists a $d$ such that each domain $D_{i}$ is a connected $n$-dimensional smooth submanifold of $\mathbb{R}^{d}$, with piecewise smooth boundary. The angle between any two intersecting smooth components of the boundary is nonzero.

Recall that a manifold is called piecewise smooth if, intuitively speaking, it is the union of finitely many smooth pieces. By smooth we will mean of class $C^{\infty}$, unless specified otherwise. Since each domain is embedded into $\mathbb{R}^{d}$, it inherits from it the standard Riemann structure so the notion of angle is defined. The non-zero angle requirement eliminates, for instance, "cusps" in dimension two, but does not eliminate "corners". Thus for domains of a hybrid system we allow disks, half-spaces, rectangles, cubes, etc. The nonzero angle assumption can be easily relaxed for most of the results, in fact it is only necessary in Section 4.

The next assumption deals with the geometry of guards.

(A3) Each guard is a smooth (n-1)-dimensional submanifold of the boundary of the corresponding domain. The boundary of each guard is piecewise smooth (or possibly empty).

There is an intuitive reasoning behind this assumption which explains why we don't allow guards to be $n$-dimensional and why we require them to be part of the boundary of domains. If $G(i, j)$ is a guard of dimension $n$, e.g., a closed set with nonempty interior in $D_{i}$, and $x(t)$ is a trajectory in the domain $D_{i}$ which meets $G(i, j)$, the corresponding execution $\chi$ jumps to $D_{j}$ as soon as $x(t)$ hits the boundary of $G(i, j)$. Therefore, the interior of $G(i, j)$ will be irrelevant for the evolution of $\chi$. If we ignore the hybrid dynamics starting in the interior of $G(i, j)$, then we can in effect remove that interior from the system as superfluous. This way we create $(n-1)$-dimensional guards which lie on the boundary of the new domains.

The following is an assumption on the resets.

(A4) Each reset is a diffeomorphism from its domain $G(e)$ onto its image. 
The image of every reset lies on the boundary of the corresponding domain. Moreover, if $e=(i, j) \in E$ and $R_{e}(p)=q$, then $X_{i}(p)=0$ if and only if $X_{j}(q)=0$.

Thus resets take equilibria to equilibria.

The next assumption deals with the relation between guards and images of resets.

(A5) Elements of $\overline{\mathcal{G}} \cup \overline{\mathcal{R}}$ (i.e., sets which are closures of guards and images of resets) can intersect only along their boundaries. Furthermore, if $p \in \bar{G} \cup \bar{R}$, then $p$ can be of only one of the following four types (Fig. 6):

Type I $p \in \operatorname{int} G \cup \operatorname{int} R$;

Type II $p \in \partial G \cup \partial R$ and there exists exactly one set $S \in \overline{\mathcal{G}} \cup \overline{\mathcal{R}}$ which contains $p$;

Type III $p \in \partial G \cup \partial R$ and there exist exactly two sets $S_{1}, S_{2} \in \overline{\mathcal{G}} \cup \overline{\mathcal{R}}$, such that $p \in \partial S_{1} \cap \partial S_{2}$ and some neighborhood of $p$ in $S_{1} \cup S_{2}$ is homeomorphic to $\mathbb{R}^{n-1}$;

Type IV $p \in \partial G \cup \partial R$ and there exist exactly two sets $S_{1}, S_{2} \in \overline{\mathcal{G}} \cup \overline{\mathcal{R}}$, such that $p \in \partial S_{1} \cap \partial S_{2}$ and some neighborhood of $p$ in $S_{1} \cup S_{2}$ is homeomorphic to $\mathbb{R}_{+}^{n-1}$.

Furthermore, resets preserve the type.

The last sentence means that if $p$ is a point of type $T \in\{I, I I, I I I, I V\}$ and $f$ is a reset map, then $f(p)$ is also of type $T$, provided that $f(p)$ is defined. Further, int and $\partial$ of $G$ and $R$ are the interior and boundary of $G$ and $R$, taken in the relative topology of these sets as subsets of the boundary of $D$. Assumption (A5) ensures that intersections of guards and images of resets (in fact, their closures) are sufficiently nice. The shaded regions in Figure 6 represent sets in $\overline{\mathcal{G}} \cup \overline{\mathcal{R}}$. They are 2-dimensional manifolds with boundary which lie on the boundary of the 3 -dimensional domain $D_{i}$. Note the following: a point $p$ of Type III lies on the boundary of two sets in $\overline{\mathcal{G}} \cup \overline{\mathcal{R}}$ and is surrounded by them from all sides; that is, there exists a neighborhood of $p$ in the union of these sets which is homeomorphic to the Euclidean space of the same dimension $(n-1)$. A point $p$ is of Type IV if it lies on the boundary of two sets in $\overline{\mathcal{G}} \cup \overline{\mathcal{R}}$, but is not surrounded by them; instead, there is a neighborhood of $p$ in the union of these sets which is homeomorphic to the Euclidean half-space of the same dimension $(n-1)$. Note that three or more objects in $\overline{\mathcal{G}} \cup \overline{\mathcal{R}}$ are not allowed to meet. We also refer the reader to the proof of Theorem 3.7 where utility of (A5) will become apparent in the construction of the hybrifold.

The following assumption deals with the vector fields along guards and images of resets. 


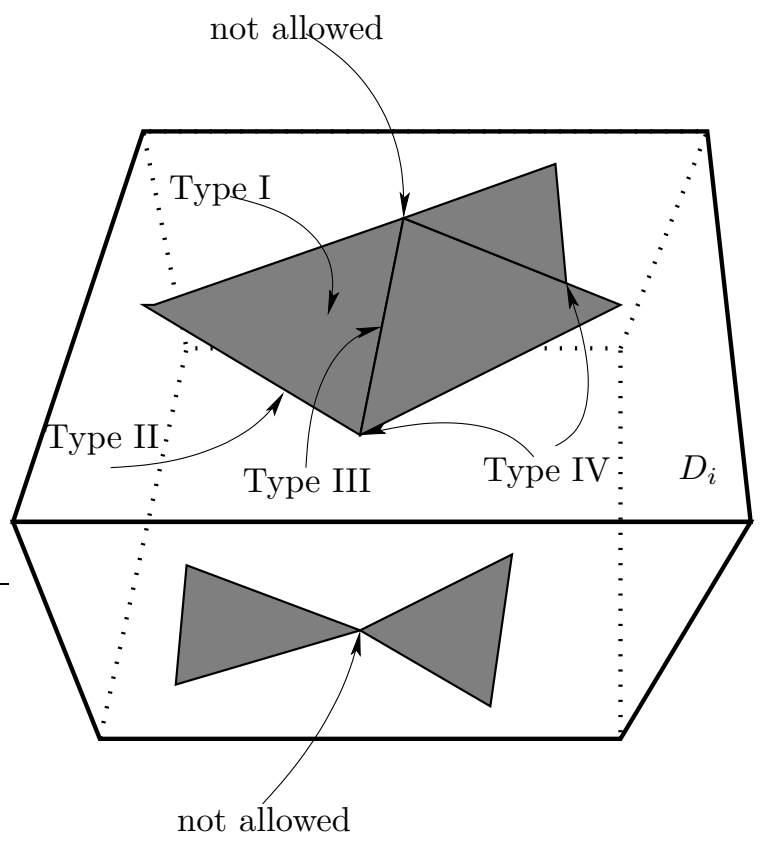

Figure 6: Types of points in (A5).

(A6) For all $e=(i, j) \in E$, the following holds: on int $G(e), X_{i}$ points outside int $D_{i}$; on $\operatorname{int}\left(\operatorname{im} R_{e}\right), X_{j}$ points inside $\bar{D}_{j}$.

Here the bar denotes closure and im the image (or range) of a map. As above, int $G(e)$ and $\operatorname{int}\left(\operatorname{im} R_{e}\right)$ denote the interior of $G(e)$ and $\operatorname{im} R_{e}$ in $\partial D_{i}$ and $\partial D_{j}$, respectively. That a vector field $X$ with flow $\phi_{t}$ points inside a set $A$ at $p \in \partial A$ means that there exists $\epsilon>0$ such that $\phi_{t}(p) \in A$, for all $0<t<\epsilon$. It points outside the same set if $-X$ points inside.

The next assumption states that resets and vector fields are smoothly extendable objects.

(A7) Each vector field $X_{i}$ is the restriction to $D_{i}$ of some smooth vector field, which we also denote by $X_{i}$, defined on a neighborhood of $D_{i}$ in $\{i\} \times \mathbb{R}^{n}$. Each reset map $R_{e}$ extends to a map $\tilde{R}_{e}$ defined on a neighborhood of $\overline{G(e)}$ in $D_{i}$ such that $\tilde{R}_{e}$ is a diffeomorphism onto its image, which is a neighborhood of im $R_{e}$ in $D_{j}$.

$F$ urthermore, suppose $f_{1}, \ldots, f_{m}, f_{m+1}:=f_{1}$ is a collection of maps such that for each $j$ there exists $e_{j} \in E$ with the property that either $f_{j}=\tilde{R}_{e_{j}}$ or $f_{j}=\tilde{R}_{e_{j}}^{-1}$, and the image of $f_{j}$ meets the domain of $f_{j+1}$. Then we have the consistency condition

$$
f_{m} \circ \cdots \circ f_{2} \circ f_{1}=\text { identity }
$$


wherever the left-hand side is defined.

The importance of this assumption is that it enables us to "glue" domains via the extended reset maps and obtain a reasonable geometric object. The condition $f_{m} \circ \cdots \circ f_{2} \circ f_{1}=$ identity simply means that a point of Type III or IV, which lies on the common boundary $C=\partial S_{1} \cap \partial S_{2}$ of some sets $S_{1}, S_{2} \in \overline{\mathcal{G}} \cup \overline{\mathcal{R}}$ will not be identified with any other point in $C$ in the gluing process. In the language of Section 3, for every $p \in C, C \cap p / \sim=\{p\}$.

The last assumption makes sure that all the above ones remain satisfied when time is reversed.

(A8) If $p \in D_{i}$ is on the boundary of $D_{i}$ and $X_{i}(p)$ points inside $D_{i}$ then $p$ is in the image of some reset.

Observe that all our examples satisfy all the assumptions above.

Definition 2.12. A hybrid system which satisfies assumptions (A1)-(A8) will be called regular.

Given $\mathbf{H}$, define a map $\Phi^{\mathbf{H}}$ on a subset of $\mathbb{R} \times D$ as follows. Let $p \in D$ be arbitrary. By (A1), there exists a unique infinite execution $(\tau, q, x)$ starting at $p$. We will denote it by $\chi(p)$. Set $\Phi^{\mathbf{H}}(0, p)=p$. Assume that $\tau_{\infty}(\chi(p))>0$. For any $0<t<\tau_{\infty}(\chi(p))$ there exist a unique $j \in\langle\tau\rangle$ such that $t \in\left[\tau_{j}, \tau_{j}^{\prime}\right)$ (even though there may be multiple $j \in\langle\tau\rangle$ for which $t \in\left[\tau_{j}, \tau_{j}^{\prime}\right]$ ). Then define

$$
\Phi^{\mathbf{H}}(t, p)=x_{j}(t) .
$$

To define $\Phi^{\mathbf{H}}(t, p)$ for negative $t$, set

$$
\Phi^{\mathbf{H}}(t, p)=\Phi^{\mathbf{H}^{\prime}}(-t, p)
$$

where $\mathbf{H}^{\prime}$ is the reverse hybrid system $\left(Q^{\prime}, E^{\prime}, \mathcal{D}^{\prime}, \mathcal{X}^{\prime}, \mathcal{G}^{\prime}, \mathcal{R}^{\prime}\right)$ defined by:

- $Q^{\prime}=Q, \mathcal{D}^{\prime}=\mathcal{D}, X_{i}^{\prime}=-X_{i}$ for all $i \in Q$;

- $(i, j) \in E^{\prime}$ if and only if $(j, i) \in E$;

- for all $e=(i, j) \in E^{\prime}, G^{\prime}(e)=R_{(j, i)}(G(j, i))$ and $R_{(i, j)}^{\prime}=R_{(j, i)}^{-1}$.

It can be checked without difficulty that $\mathbf{H}^{\prime}$ satisfies (A1)-(A8) if $\mathbf{H}$ does. Denote by $\Omega_{0}$ the largest subset of $\mathbb{R} \times D$ on which $\Phi^{\mathbf{H}}$ is defined.

In Example 2.3, for instance, for any $p \neq \mathbf{0}, \Phi^{B B}(t, p) \rightarrow \mathbf{0}$, as $t \rightarrow$ $\tau_{\infty}(\chi(p))$. Note, however, that $\chi(\mathbf{0})$ makes no time progress, i.e., $\tau_{j}=0$ for all $j \geq 0$, but it involves infinitely many switches at the same (i.e., initial) point, which happens to be a fixed point of the reset map.

Theorem 2.13. (a) $\Omega_{0}$ contains a neighborhood of $\{0\} \times \operatorname{int} D$ in $\mathbb{R} \times D$, where int $D=D-\partial D$. 
(b) For all $p \in D, \Phi^{\mathbf{H}}(0, p)=p$. Furthermore,

$$
\Phi^{\mathbf{H}}\left(t, \Phi^{\mathbf{H}}(s, p)\right)=\Phi^{\mathbf{H}}(t+s, p),
$$

whenever both sides are defined.

Proof. (a) If $p \in \operatorname{int} D_{i}$, then since $X_{i}$ is Lipschitz on $D_{i}, t \mapsto \phi_{t}^{i}(p)$ is defined on a neighborhood of 0 . Furthermore, there exists a neighborhood $U$ of $p$ in int $D_{i}$ and $\epsilon>0$ such that for each $p^{\prime} \in U, t \mapsto \phi_{t}^{i}\left(p^{\prime}\right)$ is defined on $(-\epsilon, \epsilon)$. Thus $(-\epsilon, \epsilon) \times U$ is a neighborhood of $(0, p)$ in $\mathbb{R} \times \operatorname{int} D_{i}$. This proves (a).

(b) The first statement is true by definition. The second statement follows from uniqueness of executions, guaranteed by (A1).

\section{The hybrifold and hybrid flow}

The basic idea behind the construction of the hybrifold of a hybrid system is simple: "glue" each guard to the image of the corresponding reset map. We make this more precise below and then show basic properties of the newly constructed object.

\section{The hybrifold}

Let $\mathbf{H}$ be a regular hybrid system. On $D$, let $\sim$ be the equivalence relation generated by

$$
p \sim \tilde{R}_{e}(p)
$$

for all $e \in E$ and $p \in \overline{G(e)}$. Collapse each equivalence class to a point to obtain the quotient space

$$
M_{\mathbf{H}}=D / \sim .
$$

Definition 3.1. We call $M_{\mathbf{H}}$ the hybrifold ${ }^{1}$ of $\mathbf{H}$.

Note that $M_{\mathbf{H}}$ does not depend on the choice of the extensions for the reset maps. This is because by continuity any two extensions of a fixed reset $R_{e}$ necessarily agree on $\overline{G(e)}$. Denote by $\pi$ the natural projection $D \rightarrow M_{\mathbf{H}}$ which assigns to each $p$ its equivalence class $p / \sim$. Note also that $\pi$ can easily be extended to $\bar{D}$. Put the quotient topology on $M_{\mathbf{H}}$. Recall that this is the smallest topology that makes $\pi$ continuous, i.e., a set $V \subset M_{\mathbf{H}}$ is open if and only if $\pi^{-1}(V)$ is open in $D$.

Let us apply this construction to some of the examples above.

Example 3.2 (WT continued). Without loss we assume that $l_{1}=l_{2}=0$. To obtain $M_{W T}$ we have to identify the $x_{1}$-axis from $D_{1}$ with the same axis from $D_{2}$ via $R_{(1,2)}$ and similarly with the $x_{2}$-axis (see Fig. 2 ). It is not

\footnotetext{
${ }^{1}$ The authors thank Renaud Dreyer for suggesting the term hybrifold.
} 
difficult to see that $M_{W T}$ is homeomorphic to $\mathbb{R}^{2}$ (see Fig. 7). However, $M_{W T}$ has a singularity (or "corner") at $\mathbf{0}=\pi(1,0,0)$ in the following sense. The space $D$ is equipped with a differentiable structure - the one it inherits from individual domains $D_{i}$. The space $M_{W T}$ inherits its own differentiable structure from the ambient space $\mathbb{R}^{3}$. With respect to these two differentiable structures, $\pi$ is not a smooth map. When this is the case, we will say that $\pi$ does not define a smooth structure on $M_{W T}$. As an alternative possibility, consider the standard construction of the 2-torus by identifying the opposite sides of a square (cf., Example 3.6); there, $\pi$ is a smooth map in the above sense.

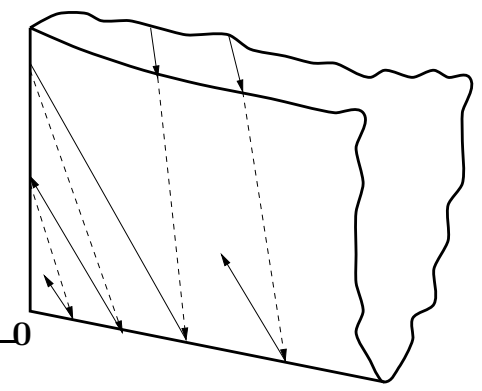

Figure 7: Hybrifold and two orbits of the hybrid flow for $W T$.

Example 3.3 ( $B B$ continued). Here we have to identify the negative part with the positive part of the $x_{2}$-axis (see Fig. 3 ). The resulting space $M_{B B}$ is again homeomorphic to $\mathbb{R}^{2}$ (see Fig. 8), but $\pi$ again does not define a smooth structure on it in the same sense as above.

Example $3.4(B B(m)$ continued). For simplicity assume $m=2$. Then we see from Fig. 9 that $M_{B B(2)}$ is smooth (in the sense that $\pi$ is a smooth map, as above) and diffeomorphic to $\mathbb{R}^{2}$.

Example 3.5 $\left(S 2(\lambda)\right.$ continued). $M_{S 2(\lambda)}$ is homeomorphic to the 2-sphere, but is not equipped with a smooth structure by $\pi$ in the sense explained above.

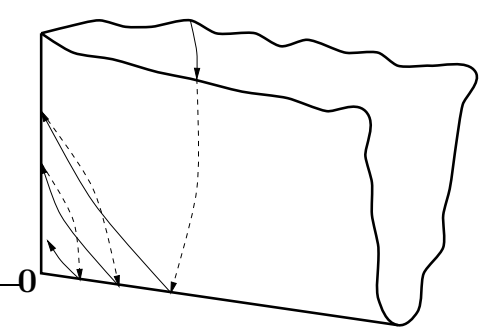

Figure 8: Hybrifold and an orbit of the hybrid flow for $B B$. 
Example 3.6 $\left(T^{2}(\alpha)\right.$ continued). We already observed that $M_{T^{2}(\alpha)}$ is the standard 2-torus.

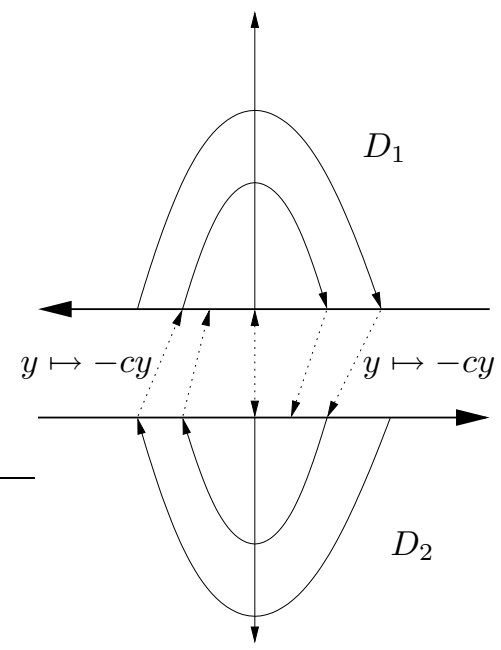

Figure 9: Hybrifold and several orbits of the hybrid flow for $B B(2)$.

The following theorem establishes some basic properties of the hybrifold. Recall that $M$ is called a topological n-manifold with boundary if it is Hausdorff and every point in $M$ has a neighborhood homeomorphic to either $\mathbb{R}^{n}$ or the closed upper half-space $\mathbb{R}_{+}^{n}=\left\{\left(x_{1}, \ldots, x_{n}\right): x_{n} \geq 0\right\}$. Points having the latter property are said to be on the boundary $\partial M$, which in itself is a topological $(n-1)$-manifold.

Theorem 3.7. (a) $M_{\mathbf{H}}$ is a topological n-manifold with boundary.

(b) Both $M_{\mathbf{H}}$ and its boundary are piecewise smooth.

(c) The restriction $\left.\pi\right|_{\text {int } D}: \operatorname{int} D \rightarrow \pi($ int $D)$ is a diffeomorphism.

Proof. (a) Recall [30] that the quotient of a manifold $M$ by an equivalence relation $\rho$ is Hausdorff if and only if the graph of $\rho$, given by $\{(p, q) \in M \times M$ : $(p, q) \in \rho\}$, is closed in $M \times M$. In our case, the relation $\rho=\sim$ is the symmetric and transitive closure of the relation $\rho_{0}$ given by

$$
\Delta_{D} \cup \bigcup_{e \in E}\left\{\left(p, \tilde{R}_{e}(p)\right): p \in \overline{G(e)}\right\},
$$

where $\Delta_{D}=\{(p, p): p \in D\}$ is the diagonal of $D \times D$. Observe that the graph of $\rho_{0}$ is a closed set. Taking the symmetric and transitive closure of $\rho_{0}$ amounts to adding to $\rho_{0}$ the graphs of all maps $f_{k} \circ \cdots \circ f_{1}$, where $f_{1}, \ldots, f_{k}$ is a collection of maps such that for some $e_{j} \in E$, either $f_{j}=\tilde{R}_{e_{j}}$ or $f_{j}=\tilde{R}_{e_{j}}^{-1}$ 
$(1 \leq j \leq k)$, and the image of $f_{j}$ meets the domain of $f_{j+1}(1 \leq j \leq k-1)$. Since the sets $\overline{G(e)}$ (the domain of $\tilde{R}_{e}$ ) and $\overline{i m R_{e}}$ (the image of $\tilde{R}_{e}$ ) are closed for all $e \in E$, and $E$ is finite, the consistency condition from (A7) guarantees that we are adding a finite number of closed sets to $\rho_{0}$. Therefore, the graph of $\sim$ is closed and $M_{\mathbf{H}}=D / \sim$ is a Hausdorff space.

Assume $x \in M_{\mathbf{H}}$. We need to show that there exists a neighborhood of $x$ in $M_{\mathbf{H}}$ which is homeomorphic to $\mathbb{R}^{n}$ or $\mathbb{R}_{+}^{n}$, where $n=\operatorname{dim} \mathbf{H}$. These neighborhoods will be the charts of the topological manifold $M_{\mathbf{H}}$.

Let $x=\pi(p)$, for some $p \in D$. If $p$ is not identified with any other points, i.e., if it is not in $\bar{G} \cup \bar{R}$, then either $p \in \operatorname{int} D$ or $p \in \partial D-(\bar{G} \cup \bar{R})$. In the former case, $p$ has a neighborhood $V$ contained in the interior of a single domain, homeomorphic to $\mathbb{R}^{n}$; since $\pi$ is one-to-one on $V, \pi(V)$ is homeomorphic to $\mathbb{R}^{n}$. In the latter case, $p$ has a neighborhood $W$ contained in a single domain, disjoint from $\bar{G} \cup \bar{R}$ and homeomorphic to $\mathbb{R}_{+}^{n}$. Since $\pi$ is one-to-one on $W$ (nothing in $W$ gets glued to anything else), $\pi(W)$ is homeomorphic to $\mathbb{R}_{+}^{n}$. This completes the case when $p$ is identified with no other points.

Before we go ahead with the proof, we state the following lemma (see Fig. 10).

Lemma 3.8 (Gluing homeomorphisms). Suppose $h_{+}: A_{+} \rightarrow \mathbb{R}_{+}^{n}$ and $h_{-}: A_{-} \rightarrow \mathbb{R}_{-}^{n}$ are homeomorphisms, where $A_{+}$and $A_{-}$are disjoint topological spaces. Let $H_{s}=h_{s}^{-1}\left(\mathbb{R}^{n-1} \times\{0\}\right)$, for $s \in\{-,+\}$, and assume that there exists a homeomorphism $g: H_{+} \rightarrow H_{-}$such that $\left.h_{+}\right|_{H_{+}}=h_{-} \circ \mathrm{g}$. Let $A=\left(A_{+} \cup A_{-}\right) / \sim$ be the quotient space, where $\sim$ is the smallest equivalence relation generated by $x \sim g(x)$, for all $x \in H_{+}$. Denote by $x / \sim$ the equivalence class of $x \in A_{+} \cup A_{-}$. Then the map $h: A \rightarrow \mathbb{R}^{n}$ defined by

$$
h(x / \sim)= \begin{cases}h_{+}(x) & \text { if } x \in A_{+} \\ h_{-}(x) & \text { if } x \in A_{-}\end{cases}
$$

is a homeomorphism.

Proof. Surjectivity, injectivity, and continuity of $h$ can be checked without difficulty. To show that $h^{-1}$ is continuous, let $U \subset A$ be an open set. Denote by $p: A_{+} \cup A_{-} \rightarrow A$ the quotient map. We will show that $V=h(U)$ is open in $\mathbb{R}^{n}$. To see that, observe first that $p^{-1}(U)=U_{+} \cup U_{-}$(disjoint union), where $U_{s}$ is open in $A_{s}, s \in\{-,+\}$ (Fig. 10). Therefore, $V_{s}=h_{s}\left(U_{s}\right)$ is open in $\mathbb{R}_{s}^{n}$. Consider an arbitrary $x \in V_{+} \cap \mathbb{R}^{n-1} \times\{0\}$. Then $h_{+}^{-1}(x) \in H_{+}$. Therefore, $x=h_{-} \circ g \circ h_{+}^{-1}(x)$. Since $g \circ h_{+}^{-1}(x) \in H_{-}$, we have that $x \in V_{-} \cap \mathbb{R}^{n-1} \times\{0\}$. Conversely, take an arbitrary $x \in V_{-} \cap \mathbb{R}^{n-1} \times\{0\}$. Then there exists $y \in H_{-}$such that $x=h_{-}(y)$. Hence, there exists $w \in H_{+}$ such that $y=g(w)$. Then $x=h_{-} \circ g(w)=h_{+}(w) \in V_{+} \cap \mathbb{R}^{n-1} \times\{0\}$. In summary, $V_{+} \cap \mathbb{R}^{n-1} \times\{0\}=V_{-} \cap \mathbb{R}^{n-1} \times\{0\}$ and hence $V=V_{-} \cup V_{+}$is open. 


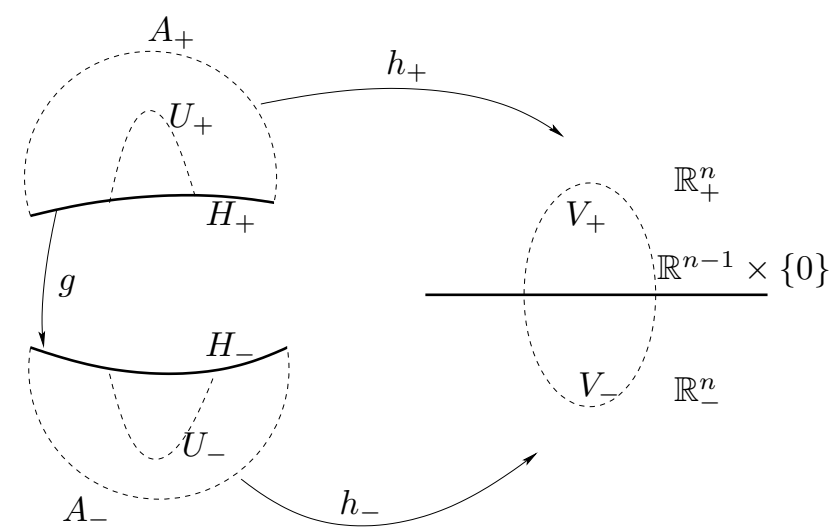

Figure 10: Gluing homeomorphisms in Lemma 3.8.

We now continue with the proof of the theorem.

If $p \in \bar{G} \cup \bar{R}$, then according to (A5) we must consider the following four cases. See Figure 11 for an illustration of Cases 1 and 2 and Figure 12 for Cases 3 and 4.

Case 1: $\mathbf{p}$ is of Type I Then there exists $e=(i, j) \in E$ such that either $p \in \operatorname{int} G(e)$ or $p \in \operatorname{int}\left(\operatorname{im} R_{e}\right)$. Without loss we can assume the former. Since interiors of sets in $\mathcal{G} \cup \mathcal{R}$ are not allowed to meet, this $e$ is unique. The point $p$ is identified with $p^{\prime}=R_{e}(p)$; note that $p / \sim=$ $\left\{p, p^{\prime}\right\}$. There exist neighborhoods $V$ (in $D_{i}$ ) and $V^{\prime}$ (in $D_{j}$ ) of $p$ and $p^{\prime}$ respectively, homeomorphic to $\mathbb{R}_{+}^{n}$ and $\mathbb{R}_{-}^{n}$ (the closed upper and lower half plane) via some homeomorphisms $h$ and $h^{\prime}$, respectively. Since $R_{e}$ is a diffeomorphism, without loss we can assume that $\left.h\right|_{V \cap G(e)}=$ $\left.h^{\prime} \circ R_{e}\right|_{V \cap G(e)} .^{2}$ Then, by Lemma 3.8, $U=\pi\left(V \cup V^{\prime}\right)$ is homeomorphic to $\mathbb{R}^{n}$ via a homeomorphism $h_{U}$. Note that $h_{U}$ is, in fact, piecewise smooth. Thus $x \in \operatorname{int} M_{\mathbf{H}}$ and $\left(U, h_{U}\right)$ is a chart of $M_{\mathbf{H}}$ at $x$.

Case 2: $p$ is of Type II Then $p$ is on the boundary of exactly one set $S \in$ $\overline{\mathcal{G}} \cup \overline{\mathcal{R}}$. Without loss we may assume $S=\overline{G(e)}$, for a unique $e=$ $(i, j) \in E$. Hence $p$ gets identified with (a unique point) $p^{\prime}=\tilde{R}_{e}(p) \in$ $\partial\left(\operatorname{im} R_{e}\right)$; observe that $p / \sim=\left\{p, p^{\prime}\right\}$. There exist neighborhoods $V$ (in $D_{i}$ ) and $V^{\prime}$ (in $D_{j}$ ) of $p$ and $p^{\prime}$, homeomorphic to $\mathbb{R}_{+}^{n}$ and $\mathbb{R}_{-}^{n}$ via some homeomorphisms $h$ and $h^{\prime}$, respectively. Note that only a proper subset of the boundary of $V$ (namely, $V \cap \overline{G(e)}$ ) is identified with a proper subset of the boundary of $V^{\prime}$ (namely, $V^{\prime} \cap \overline{\mathrm{im} R_{e}}$ ). Since $\tilde{R}_{e}$ is a diffeomorphism, without loss we can assume that $\left.h\right|_{V \cap \overline{G(e)}}=$

\footnotetext{
${ }^{2}$ If not, instead of $h$ consider the homeomorphism $\hat{h}: V \rightarrow \mathbb{R}_{+}^{n}$ defined by $\hat{h}=F \circ h$, where $F: \mathbb{R}_{+}^{n} \rightarrow \mathbb{R}_{+}^{n}$ is any homeomorphism which extends $h^{\prime} \circ R_{e} \circ\left(\left.h\right|_{V \cap G(e)}\right)^{-1}$ : $\mathbb{R}^{n-1} \times\{0\} \rightarrow \mathbb{R}^{n-1} \times\{0\}$. Then it is easy to check that $\left.\hat{h}\right|_{V \cap G(e)}=\left.h^{\prime} \circ R_{e}\right|_{V \cap G(e)}$.
} 
$\left.h^{\prime} \circ \tilde{R}_{e}\right|_{V \cap \overline{G(e)}}$. By a construction analogous to the one in Lemma 3.8, it follows that $U=\pi\left(V \cup V^{\prime}\right)$ is homeomorphic to $\mathbb{R}_{+}^{n}$ via some $h_{U}$. Thus $x \in \partial M_{\mathbf{H}}$ and $\left(U, h_{U}\right)$ is a chart of $M_{\mathbf{H}}$ at $x$. Observe that the restriction of $h_{U}$ is a piecewise smooth homeomorphism between $U \cap \partial M_{\mathbf{H}}$ and $\mathbb{R}^{n-1}$. Therefore, $\left(U \cap \partial M_{\mathbf{H}},\left.h_{U}\right|_{U \cap \partial M_{\mathbf{H}}}\right)$ is a chart for the boundary of $M_{\mathbf{H}}$.

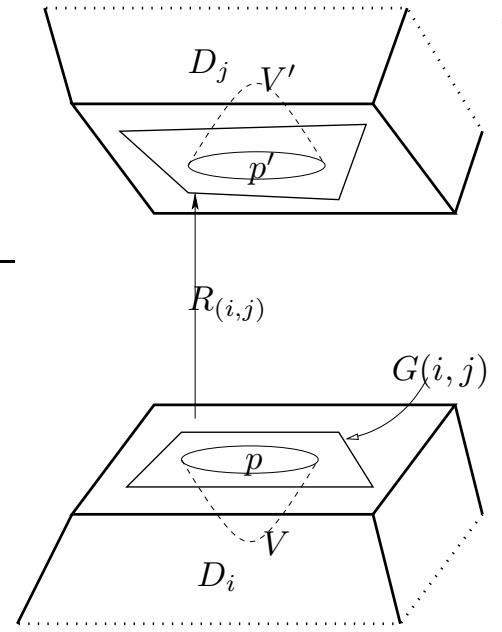

Case 1

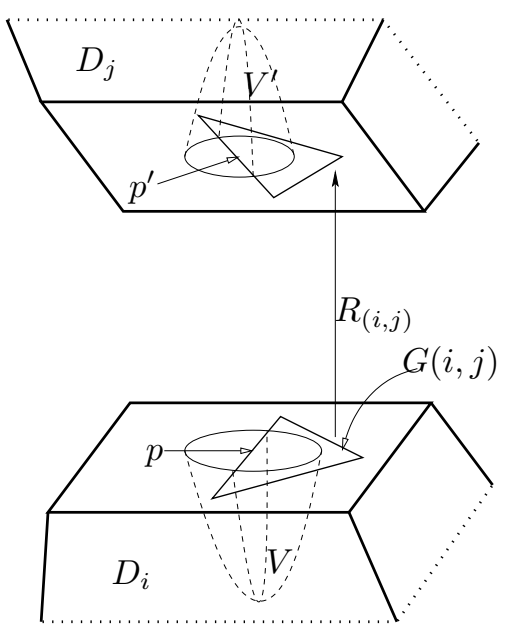

Case 2

Figure 11: Proof of Theorem 3.7(a), Cases 1 and 2.

Case 3: $p$ is of Type III Assume $p / \sim=\left\{p_{1}, \ldots, p_{m}\right\}$, where $p_{1}=p$ and $p_{j} \in D_{i_{j}}$. For each $j$, there exist sets $S_{1}^{j}, S_{2}^{j} \in \overline{\mathcal{G}} \cup \overline{\mathcal{R}}$ such that $p_{j} \in$ $\partial S_{1}^{j} \cap \partial S_{2}^{j}$. Further, there exist maps $f_{1}, \ldots, f_{m}$ such that for each $j$, $f_{j}$ is either $\tilde{R}_{e_{j}}$ or $\tilde{R}_{e_{j}}^{-1}$, for some $e_{j} \in E$, and $f_{j}$ maps $S_{2}^{j}$ onto one of the sets $S_{1}^{1}, S_{2}^{1}, \ldots, S_{1}^{m}, S_{2}^{m}$ other than $S_{2}^{j}$. Without loss of generality, we can assume that

$$
f_{j}: S_{2}^{j} \rightarrow S_{1}^{j+1}
$$

where $S_{1}^{m+1}=S_{1}^{1}, S_{2}^{m+1}=S_{2}^{1}$. See Fig. 12 (where $n=3, m=3$ ). Since $p_{j}$ is of Type III, there exists a neighborhood $V_{j}$ of $p_{j}$ in $S_{1}^{j} \cup S_{2}^{j}$, such that $V_{j}$ is homeomorphic to $\mathbb{R}^{n-1}$. We require that $V_{j} \cap \partial S_{i}^{j}(i=1,2$, $1 \leq j \leq m$ ) be a neighborhood of $p_{j}$ in $\partial S_{i}^{j}$ (with respect to the relative topology $\partial S_{i}^{j}$ inherits from $S_{i}^{j}$ ). By the consistency condition (2) of (A7), it is possible to choose $V_{1}, \ldots, V_{m}$ so that for all $j$,

$$
f_{j}\left(S_{2}^{j} \cap V_{j}\right)=S_{1}^{j+1} \cap V_{j+1} .
$$

This means that $\pi\left(V_{1}\right)=\cdots=\pi\left(V_{m}\right)$. 
Furthermore, for each $j$, there exists a set $U_{j}$ open in $D_{i_{j}}$, such that $U_{j}$ is homeomorphic to $\mathbb{R}_{+}^{n}$ and $V_{j}=U_{j} \cap \partial D_{i_{j}}$. Let $U=\pi\left(U_{1} \cup \cdots \cup U_{m}\right)$; this set is obtained by gluing $U_{1}, \ldots, U_{m}$ along $V_{1}, \ldots, V_{m}$ via the maps $f_{1}, \ldots, f_{m}$. It follows by construction that $U$ is a neighborhood of $x=\pi\left(p_{1}\right)$ in $M_{\mathbf{H}}$ and that $U$ is homeomorphic to $\mathbb{R}^{n}$. This in particular means that $p$ is in the interior of $M_{\mathbf{H}}$ and $U$ is a chart of $M_{\mathbf{H}}$ as a topological manifold. This completes Case 3.

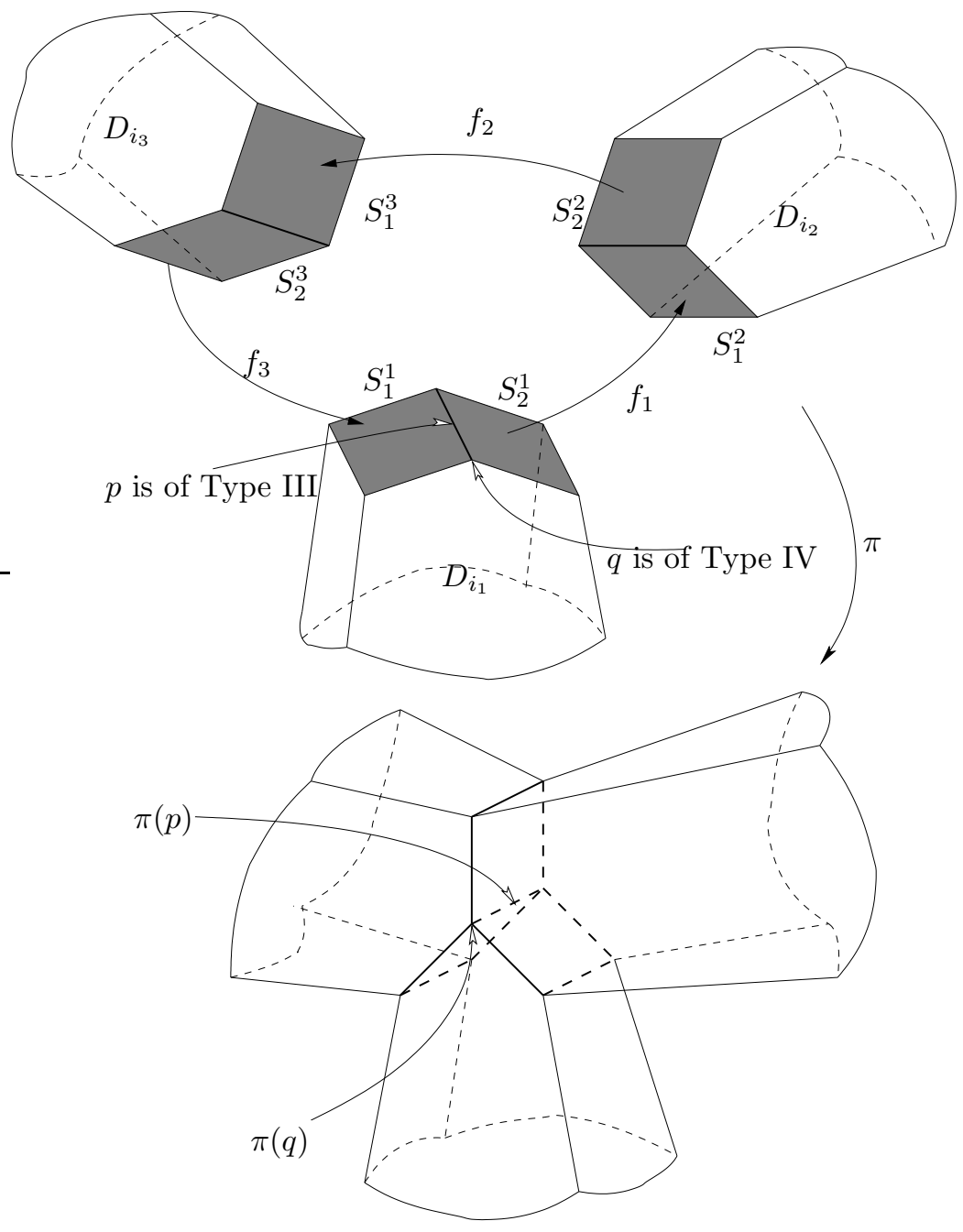

Figure 12: Proof of Theorem 3.7(a), Cases 3 and 4.

Case 4: $p$ is of Type IV This proof is similar to the previous one. Consider the point $q$ of Type IV in Fig. 12. Analogously as in the proof 
of Case 3, we obtain sets $S_{1}^{1}, S_{2}^{1}, \ldots, S_{1}^{m}, S_{2}^{m} \in \overline{\mathcal{G}} \cup \overline{\mathcal{R}}, U_{1}, \ldots, U_{m}$, and $V_{1}, \ldots, V_{m}$ such that: $(i) U_{j}$ is open in $D_{i_{j}}$ and homeomorphic to $\mathbb{R}_{+}^{n} ;($ ii $) V_{j}$ is a neighborhood of $p_{j}$ in $S_{1}^{j} \cup S_{2}^{j}$ homeomorphic to $\mathbb{R}_{+}^{n-1} ;$ (iii) $V_{j}=U_{j} \cap \partial D_{i_{j}}$; and $(i v) \pi\left(V_{1}\right)=\cdots=\pi\left(V_{m}\right)$. Then $U=\pi\left(U_{1} \cup \cdots \cup U_{m}\right)$ is homeomorphic to $\mathbb{R}_{+}^{n}$ via a piecewise smooth homeomorphism. Furthermore, $y=\pi(q)$ lies on the boundary of $M_{\mathbf{H}}$ and $U$ is a chart of $M_{\mathbf{H}}$ at $y$; also, $U \cap \partial M_{\mathbf{H}}$ is a neighborhood of $y$ in $\partial M_{\mathbf{H}}$, homeomorphic (via a piecewise smooth homeomorphism) to $\mathbb{R}_{+}^{n-1}$, hence a chart of $\partial M_{\mathbf{H}}$ at $y$.

(b) Piecewise smoothness of $M_{\mathbf{H}}$ and its boundary follow from piecewise smoothness of their charts introduced in (a).

(c) A direct consequence of the above construction.

Remark 2. It is clear that the hybrifold can be a very complicated object, especially in dimensions greater than two (consider, for instance, $\left.M_{B B S(N)}\right)$. The complexity of the hybrifold is simply the reflection of the complexity of the original hybrid system; it is a measure of how complicated the interplay is between the discrete-time and continuous-time dynamics in the original hybrid system.

\section{Remark on metrizability}

We point out that every hybrifold $M_{\mathbf{H}}$ is metrizable, that is, it admits a metric $d_{\mathbf{H}}: M_{\mathbf{H}} \times M_{\mathbf{H}} \rightarrow \mathbb{R}_{+}$which generates the already existing (quotient) topology on $M_{\mathbf{H}}$. This follows from Urysohn's metrization theorem (every regular space with a countable basis is metrizable) and from the just proven fact that $M_{\mathbf{H}}$ is a manifold. See for example, [30]. Observe, however, that if $D$ is given a metric space structure, in general it may not be possible to define $d_{\mathbf{H}}$ so that the projection $\pi$ is an isometry - a quotient of a metric space may not even be metrizable. Also note that the usual definition of distance between points as on a Riemannian manifold may not work either. Since metrizability is not used in the rest of the paper, we will not discuss this point any further.

\section{The hybrid flow}

Define the hybrid flow $\Psi^{\mathbf{H}}$ by

$$
\Psi^{\mathbf{H}}(t, \pi(p))=\pi \Phi^{\mathbf{H}}(t, p) .
$$

This possibly set-valued map is defined on

$$
\Omega=\left\{(t, \pi(p)):(t, p) \in \Omega_{0}\right\} .
$$


Orbits of $\Psi^{\mathbf{H}}$ are obtained by projecting orbits of $\Phi^{\mathbf{H}}$ by $\pi$. By the $\Phi^{\mathbf{H}}$-orbit of $p$ we mean the collection of points $\Phi^{\mathbf{H}}(t, p)$ for all $t$ such that $(t, p) \in \Omega_{0}$.

Example 3.9. Consider the examples from the previous subsection. Observe that if $\mathbf{H}=W T, B B$ or $B B(m)$, then for all $x \in M_{\mathbf{H}}$, we have $\Psi^{\mathbf{H}}(t, x) \rightarrow$ $\pi(\mathbf{0})$, as $t \rightarrow \tau_{\infty}(\chi(x))$. In other words, all executions "spiral" towards zero. If $\mathbf{H}=S 2(\lambda)$, then the hybrid flow has two equilibria corresponding to the saddles in the original system and four "Zeno states", that is, points at which trajectories accumulate in a finite amount of time. The hybrid flow of $\Psi^{T^{2}}(\alpha)$ is just the linear flow on the 2 -torus. If $\alpha$ is rational, every orbit is closed; if it is irrational, every orbit is dense in the torus.

Fix $\mathbf{H}$ and set $\Psi:=\Psi^{\mathbf{H}}$. For each $t \in \mathbb{R}$ and $x \in M_{\mathbf{H}}$, let

$$
M(t)=\left\{y \in M_{\mathbf{H}}: \Psi(t, y) \text { is defined }\right\},
$$

and

$$
J(x)=\{s \in \mathbb{R}: \Psi(s, x) \text { is defined }\} .
$$

Observe that if $x=\pi(p)$, then $J(x) \cap[0, \infty)=\left[0, \tau_{\infty}(\chi(p))\right)$. Also, for $t>0$, $M(t)$ contains all points $x=\pi(p)$ such that $\tau_{\infty}(\chi(p))>t$. As usual, $\chi(p)$ denotes the unique execution of $\mathbf{H}$ starting at $p$.

If $M(t)$ is not empty, denote by $\Psi_{t}$ the time $t$ map of $\Psi$, defined by

$$
\Psi_{t}(x)=\Psi(t, x) \text {. }
$$

Note again that the time $t$ map of the hybrid flow of a regular hybrid system may be multi-valued. This occurs when, due to gluing, the projections of two disjoint executions of $\mathbf{H}$ overlap in the hybrifold. This can happen at $x=\pi(p) \in M_{\mathbf{H}}$, where $p$ is a point of type III or IV (cf. (A5)); since $p$ is glued to two or more other points, the orbit through $x$ could branch. We will deal with this situation shortly.

Definition 3.10. Let $X$ be a smooth vector field on a smooth manifold $M$, with flow $\phi_{t}$. We say that $q \in M$ is $X$-reachable from a point $q \in M$, if $q=\phi_{t}(p)$, for some $t>0$. A set $S$ is $X$-reachable from a point $p$ if there exists a point $q \in S$ such that $q$ is $X$-reachable from $p$.

To avoid the "branching scenario" described above we now introduce the following property of hybrid systems.

Definition 3.11. A regular hybrid system $\mathbf{H}$ is said to be without branching if for every point $p \in \partial D$ of type III or IV with $p / \sim=\left\{p_{1}, \ldots, p_{m}\right\}$, where $p_{j} \in D_{i_{j}}$, there exists at most one $k$ and at most one $\ell, 1 \leq k \neq \ell \leq m$, such that $p_{k}$ is $X_{i_{k}}$-reachable from $D_{i_{k}}$ and $D_{i_{\ell}}$ is $X_{i_{\ell}}$-reachable from $p_{\ell}$.

From this point on, all hybrid systems in this paper will be assumed to be regular and without branching. Note that all our examples have these properties. Observe also that $\mathbf{H}$ is without branching if and only if the reverse hybrid system $\mathbf{H}^{\prime}$ is. We can now state the following theorem. 
Theorem 3.12. Suppose that $\mathbf{H}$ is regular and without branching. Then:

(a) For each $(t, x) \in \Omega, \Psi_{t}(x)$ is a single point.

(b) For each $x \in M_{\mathbf{H}}$, the map $t \mapsto \Psi_{t}(x)$ is continuous. Moreover, if $J(x)$ is not a single point, the map is smooth except at (at most) countably many points in $J(x)$.

(c) Each map $\Psi_{t}$ is one-to-one.

(d) Whenever both sides are defined,

$$
\Psi_{t} \Psi_{s}(x)=\Psi_{t+s}(x)
$$

(e) For each $t \in \mathbb{R}$, there exists an open and dense subset of $M(t)$ on which $\Psi_{t}$ is continuous.

Proof. (a) Nonuniqueness of executions in $M_{\mathbf{H}}$ can occur only at points $x=\pi(p) \in M_{\mathbf{H}}$ such that $p$ is of Type III or IV, and more than one trajectory of $\Psi$ enters or leaves $x$. This situation is eliminated by the no branching condition.

(b) Let $x=\pi(p)$ for some $p \in D$. Let $\chi(p)=(\tau, q, x)$ be the unique execution of $\mathbf{H}$ starting at $p$ (i.e., $\tau_{0}=0$ and $x_{0}(0)=p$ ). Recall that for positive $t$, $\Psi_{t}(x)=\pi \Phi^{\mathbf{H}}(t, p)=\pi x_{j}(t)$, if $t \in\left[\tau_{j}, \tau_{j}^{\prime}\right)$. Thus it is enough to check continuity of $t \mapsto \Psi_{t}(x)$ at $\tau_{j}^{\prime}=\tau_{j+1}$, for $j \geq 0$. But recall that $t \mapsto \Phi^{\mathbf{H}}(t, p)$ is continuous from the right, with discontinuities of the first kind only at $\tau_{j}$, $j \geq 0$. Since $x_{j}\left(\tau_{j}\right) \in G$ and

$$
\pi\left(x_{j}\left(\tau_{j}^{\prime}\right)\right)=\pi\left(x_{j+1}\left(\tau_{j+1}\right)\right),
$$

for all $j \geq 0$, it follows that

$$
\lim _{t \rightarrow \tau_{j}^{\prime}-} \Psi_{t}(x)=\lim _{t \rightarrow \tau_{j}^{\prime}-} \pi x_{j}(t)=\pi x_{j+1}\left(\tau_{j+1}\right)=\Psi_{\tau_{j}^{\prime}}(x),
$$

which shows that $t \mapsto \Psi_{t}(x)$ is continuous on $J(x) \cap[0, \infty)$.

Continuity of $t \mapsto \Psi_{t}(x)$ for negative $t$ follows by observing that $\Psi_{-t}^{\mathbf{H}}(x)=$ $\Psi_{t}^{\mathbf{H}^{\prime}}(x) \quad(t>0)$, where $\mathbf{H}^{\prime}$ is the reverse hybrid system to $\mathbf{H}$.

The extreme case when $J(x)=\{0\}$ happens when $x=\pi(p)$ for some $p \in D$ such that:

$$
p \in \overline{G\left(e_{0}\right)}, p_{1}=\tilde{R}_{e_{0}}(p) \in \overline{G\left(e_{1}\right)}, p_{2}=\tilde{R}_{e_{1}}\left(p_{1}\right) \in \overline{G\left(e_{2}\right)}, \text { etc. }
$$

for a sequence $e_{0}, e_{1}, e_{2}, \ldots$ in $E$. Then $t \mapsto \Psi_{t}(x)$ is trivially continuous.

Assume now that $J(x)$ is not a single point. With the notation as above, we have that for $\tau_{j}<t<\tau_{j}^{\prime}$,

$$
\frac{d}{d t} \Psi_{t}(x)=T \pi\left(X_{q(j)}\left(x_{j}(t)\right)\right),
$$


which proves that $t \mapsto \Psi_{t}(x)$ is piecewise smooth. Here $T \pi$ denotes the tangent (or derivative) map of $\pi$. It is defined at $x_{j}(t)$ because $x_{j}(t) \in \operatorname{int} D$, for $\tau_{j}<t<\tau_{j}^{\prime}$, and $\pi$ is smooth on int $D$.

(c) Injectivity of $\Psi_{t}$ follows directly from uniqueness of executions through any point.

(d) Follows from (a) and the analogous property of $\Phi^{\mathbf{H}}$.

(e) It is enough to show this for $t>0$ with $M(t) \neq \emptyset$. For $x \in M(t)$, denote by $\mathcal{O}_{[0, t]}(x)$ the set of points $\Psi_{s}(x)$, for all $0 \leq s \leq t$. Let

$$
M_{d}(t)=\left\{x \in M(t): \mathcal{O}_{[0, t]}(x) \text { intersects } \pi(\partial G)\right\},
$$

and $M_{c}(t)=M(t)-M_{d}(t)$. So $M_{d}(t)$ is the set of all points in $M(t)$ such that on the time interval $[0, t]$ their $\Psi$-orbits pass through the projection of the boundary of some guard (and thus the boundary of the image of some reset). On the other hand, the $[0, t]$-segment of the orbit of any point in $M_{c}(t)$ passes only through the projection of interiors of guards or is possibly trapped in some domain.

It is not difficult to see that $M_{c}(t)$ is open and dense in $M(t)$. Furthermore, we claim that $\Psi_{t}$ is continuous on $M_{c}(t)$. To see this, let $x_{0} \in M_{c}(t)$ be arbitrary and let $y_{0}=\Psi_{t}\left(x_{0}\right)$. Let $N$ be the number of guards which $\mathcal{O}_{[0, t]}\left(x_{0}\right)$ intersects. We prove that $\Psi_{t}$ is continuous at $x_{0}$ by induction on $N$. If $N=0$, we are dealing with a flow of a single vector field on an invariant set, so the result follows from standard theory of differential equations. If $N>0$, since continuity is preserved under composition, it suffices to treat the case $N=1$. So let $N=1$ and let $V$ be a neighborhood of $y_{0}$ in $M_{\mathbf{H}}$. Assume $x_{0} \in \pi\left(D_{i}\right)$ and $y_{0} \in \pi\left(D_{j}\right)$. Let $w_{0}$ be the intersection of $\mathcal{O}_{[0, t]}\left(x_{0}\right)$ with the only guard (more precisely, its projection by $\pi$ ) it intersects, namely, $\Gamma=\pi(G(i, j))$. Then $w_{0} \in \operatorname{int} \Gamma$, and $w_{0}=\Psi_{s}\left(x_{0}\right)$, for some $0 \leq s \leq t$ (see Fig. 13). We assume that $0<s<t$; other cases are treated analogously. Then, there exists a neighborhood $W \subset \Gamma$ of $w_{0}$ such that for every $w \in W$, there exist $r$ with $0<r<t$ such that $\Psi_{r}(w) \in V$. This follows from continuity of the flow of $X_{j}$. Since the flow of $X_{i}$ is also continuous, there exists a neighborhood $U$ of $x_{0}$ such that for all $x \in U, \mathcal{O}_{[0, t]}(x)$ passes through $W$. By taking a smaller $U$ if necessary, we get that for every $x \in U, \Psi_{t}(x) \in V$. Therefore, $\Psi_{t}$ is continuous at $x_{0}$. Observe that $\Psi$ may well be continuous also on $M_{d}(t)$ (e.g., Example 2.7). Compare also [36].

Remark 3. For $x \in M_{\mathbf{H}}$ with $x=\pi(p)$, we set $\chi(x)=\pi(\chi(p))$ and refer to it as the execution (in $M_{\mathbf{H}}$ ) starting from $x$. Furthermore, properties of $\chi(p)$ transfer to properties of $\chi(x)$, e.g., we say that $\chi(x)$ is a Zeno execution if $\chi(p)$ is, and so on. 


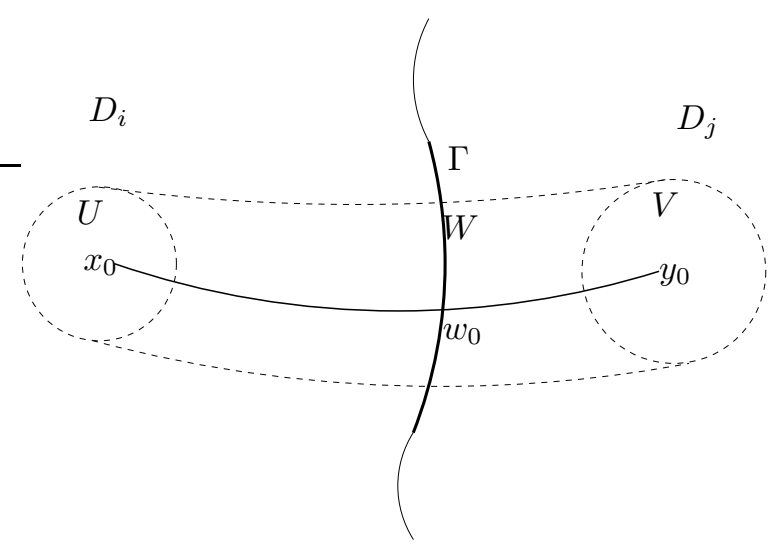

Figure 13: Proof of Theorem 3.12(e).

\section{$4 \omega$-limit sets and the Zeno phenomenon}

Zeno executions do not arise in physical systems, but are a consequence of modeling over-abstraction. However, as we have seen, it is easy to construct seemingly realistic systems which admit Zeno behavior. Therefore, one would like to be able to identify and eliminate it. In this section we deal with the first problem. From a mathematical viewpoint, the Zeno phenomenon poses several interesting questions: for instance, what is its topological cause? Is there a checkable criterion which guarantees the non-occurence of Zeno? How should the original system be modified to remove Zeno executions? In this section we show that, in short, the topological cause of Zenoness is lack of smoothness in the hybrid flow.

Since we would like to study the long term behavior of executions of hybrid systems, we define the following notion.

Definition 4.1. A point $y \in M_{\mathbf{H}}$ is called an $\omega$-limit point of $x \in M_{\mathbf{H}}$ if

$$
y=\lim _{m \rightarrow \infty} \Psi_{t_{m}}^{\mathbf{H}}(x),
$$

for some increasing sequence $\left(t_{m}\right)$ in $J(x)$ such that $t_{m} \rightarrow \tau_{\infty}(x)$, as $m \rightarrow \infty$. The set of all $\omega$-limit points of $x$ is called the $\omega$-limit set of $x$ and is denoted by $\omega(x)$.

In other words, $\omega$-limit points for $x$ are accumulation points of the orbit of $x$. By $\tau_{\infty}(x)$ we denote the execution time of the unique infinite execution of $\mathbf{H}$ starting from some $p$, where $x=\pi(p)$; that is, $\tau_{\infty}(x)=\tau_{\infty}(\chi(p))$. It is easy to check that this is a well defined element of $[0, \infty]$.

Suppose $x \in M_{\mathbf{H}}$ and denote by $E_{\infty}(x) \subset E$ the set of discrete transitions which occur infinitely many times in the execution starting from $x$. If $E_{\infty}(x)$ 
is empty, then the orbit of $x$ eventually ends up in a single domain $D_{i}$ (that is, its image under $\pi$ in the hybrifold) in which case

$$
\omega(x) \subset \pi\left(\overline{D_{i}}\right) .
$$

This means that every point $y \in \omega(x)$ is an accumulation point of the orbit of a single vector field, namely $X_{i}$. If $E_{\infty}(x)$ is non-empty, but $\tau_{i}=\tau_{\infty}(x)$ for some $i \in\langle\tau\rangle$, then every point $y \in \omega(x)$ is an accumulation point of the orbit of a discrete-time system generated by the resets $R_{e}, e \in E_{\infty}(x)$. We will call points $y \in \omega(x)$ that involve only the continuous or only the discrete dynamics pure $\omega$-limit points.

If $E_{\infty}(x)$ is nonempty and $\tau_{i}<\tau_{\infty}(x)$ for all $i \in\langle\tau\rangle$, then every $\omega$ limit point for $x$ is a result of both the continuous and discrete (i.e., hybrid) dynamics of $\mathbf{H}$ and will accordingly be called a hybrid $\omega$-limit point of $x$.

A special case of a hybrid $\omega$-limit point is a Zeno state:

Definition 4.2. A point $z \in M_{\mathbf{H}}$ is called a Zeno state for $x$ if $z \in \omega(x)$ and $\chi(x)$ is a dynamic Zeno execution.

In other words, a Zeno state is an accumulation point of a dynamic Zeno execution. (Recall that a Zeno execution is called dynamic if $\tau_{j} \neq \tau_{j}^{\prime}$ for infinitely many $j$ 's.) We will also refer to points in $\pi^{-1}(z)$ as Zeno states in H. For example, the "origin" $\mathbf{0}$ of $M_{W T}$ (as well as $M_{B B}$ and $M_{B B(2)}$ ) is a Zeno state for every point different from $\mathbf{0}$. Moreover, for each $x \neq \mathbf{0}, \omega(x)$ contains only one Zeno state. We now show this is always the case.

Proposition 4.3. If the execution starting from $x \in M_{\mathbf{H}}$ is Zeno and bounded, then $\omega(x)$ consists of exactly one Zeno state for $x$. Moreover,

$$
\omega(x) \subset \bigcap_{e \in E_{\infty}(x)} \pi(\overline{G(e)})=\bigcap_{e \in E_{\infty}(x)} \pi(\partial G(e)) .
$$

Proof. Let $p \in \pi^{-1}(x)$ be arbitrary and, as before, let $\chi(p)=(\tau, q, x)$ be the unique execution starting from $p$. For $j \geq 0$, let $A_{j}=\pi\left(x_{j}\left(I_{j}\right)\right)$. Then $A_{j}$ is an arc in $\pi\left(D_{q(j)}\right)$ of the $\Psi^{\mathbf{H}}$-orbit of $x$. Since the set $A=\bigcup A_{j}$ is by assumption bounded, it must have an accumulation point, say $z$. Clearly, $z \in \omega(x)$ and $z$ is a Zeno state for $x$. Furthermore, all vector fields $X_{i}$ are bounded on $A=\bigcup A_{j}$. (This is true, for example, if the reset maps are non-expanding; see $[41,42]$.) Therefore,

$$
\sum_{j=0}^{\infty}\left|A_{j}\right|<\infty
$$

where $\left|A_{j}\right|$ is the length of $x_{j}\left(I_{j}\right)$ as a curve in $\mathbb{R}^{d}$.

Suppose there exists another accumulation point of $A$ or equivalently, another Zeno state $z^{\prime}$ for $x$. Then we have:

$$
z=\lim _{m \rightarrow \infty} \Psi_{t_{m}}^{\mathbf{H}}(x), \quad z^{\prime}=\lim _{m \rightarrow \infty} \Psi_{t_{m}^{\prime}}^{\mathbf{H}}(x),
$$


for some increasing sequences $\left(t_{m}\right)$ and $\left(t_{m}^{\prime}\right)$ in $J(x)$ such that $t_{m}, t_{m}^{\prime} \rightarrow$ $\tau_{\infty}(x)$, as $m \rightarrow \infty$. If

$$
x_{m}=\Psi_{t_{m}}^{\mathbf{H}}(x) \in A_{j_{m}} \quad \text { and } \quad x_{m}^{\prime}=\Psi_{t_{m}^{\prime}}^{\mathbf{H}}(x) \in A_{j_{m}^{\prime}},
$$

for some $j_{m}, j_{m}^{\prime} \rightarrow \infty$, we obtain

$$
\left|x_{m} x_{m}^{\prime}\right| \leq \sum_{l=\min \left(j_{m}, j_{m}^{\prime}\right)}^{\infty}\left|A_{l}\right| \rightarrow 0
$$

as $m \rightarrow \infty$, where $\left|x_{m} x_{m}^{\prime}\right|$ denotes the length of the segment of $A$ between $x_{m}$ and $x_{m}^{\prime}$. Thus $z=z^{\prime}$. This completes the first part of the proof.

To show (3), let $\omega(x)=\{z\}$, and let $e=(i, j) \in E_{\infty}(x)$. Then there exists a sequence $\left(x_{m}\right)$ of points in $D_{i}$ such that: $x_{m}$ is on the forward orbit of $x$ and $x_{m} \rightarrow z$ as $m \rightarrow \infty$. Thus: $z \in \pi\left(\overline{D_{i}}\right)$. Similarly, $z \in \pi\left(\overline{D_{j}}\right)$. But $\pi\left(\overline{D_{i}}\right) \cap \pi\left(\overline{D_{j}}\right) \subset \pi(\overline{G(e)})$, so $z \in \pi(\overline{G(e)})$. Since this holds for all $e \in E_{\infty}(x)$, the proof of (3) is complete.

Remark 4. Accumulation points of static Zeno executions are called chattering states. So a Zeno state is a hybrid $\omega$-limit point whereas a chattering state is a pure discrete $\omega$-limit point. Note that a chattering state for one execution may be a Zeno state for another. For instance, in $M_{W T}$ and $M_{B B}$, $\mathbf{0}$ is a chattering state for $\chi(\mathbf{0})$, but a Zeno state for $\chi(x)$, for every $x \neq \mathbf{0}$.

Note that in all the Zeno examples above none of the flows involved in creating the Zeno state has an equilibrium at the Zeno state. (This is not necessarily the case for purely chattering states.) The following lemma shows that this is not a coincidence.

Proposition 4.4. A Zeno state is not an equilibrium of any of the vector fields that participate in creating it. More precisely, if $z \in M_{\mathbf{H}}$ is a Zeno state for $x$, then for every $p \in \pi^{-1}(z)$, if $p \in \overline{D_{i}}$ and $D_{i}$ is visited infinitely often by the lift by $\pi$ of the orbit of $x$, then $X_{i}(p) \neq 0$.

Proof. Let $z$ be a Zeno state for $x$. Consider the lift of the orbit of $x$ by $\pi$ to $D$ and let us concentrate on its "trace" in a particular domain which it visits infinitely often.

More precisely, there exist $j \in Q, e=(i, j), e^{\prime}=\left(j, i^{\prime}\right) \in E$ and $p_{*} \in D_{j}$ such that $\pi\left(p_{*}\right)=z$ and

$$
p_{*} \in \overline{\operatorname{im} R_{e}} \cap \overline{G\left(e^{\prime}\right)} .
$$

Furthermore, there exists a sequence $\left(p_{m}\right)$ in im $R_{e}$ converging to $p_{*}$, and a sequence $\left(t_{m}\right)$ of positive numbers such that (see Fig. 14)

$$
q_{m}=\phi_{t_{m}}^{j}\left(p_{m}\right) \in G\left(e^{\prime}\right) \quad \text { and } \quad T_{\infty}:=\sum_{m=0}^{\infty} t_{m}<\infty
$$


Recall that $\left\{\phi_{t}^{j}\right\}$ is the (local) flow of the vector field $X:=X_{j}$ on $D_{j}$.

Let $A_{m}$ be the arc tangent to $X$ and connecting $p_{m}$ and $q_{m}$ (i.e., $A_{m}$ is the $X$-orbit of $\left.p_{m}\right)$, and denote by $\left|A_{m}\right|$ its length. Finally, let $K \subset D_{j}$ be a compact set containing $p_{*}$ and $A_{m}$ for all $m \geq 0$. It exists, because $p_{m} \rightarrow p_{*}$, as $m \rightarrow \infty$.

The intuition is as follows: we start from $p_{0}$, flow for time $t_{0}$ to $q_{0}$ when we reach the guard $G\left(e^{\prime}\right)$ and are taken outside of $D_{j}$ by a reset. We enter $D_{j}$ again at $p_{1}$, flow for time $t_{1}$ until we reach $q_{1}$, etc.

Now assume that $p_{*}$ is an equilibrium for $X$. Then $\phi_{t}^{j}\left(p_{*}\right)=p_{*}$ for all $t \in \mathbb{R}$. If $p \in A_{m}$, then $p=\phi_{t}^{j}\left(p_{m}\right)$ for some $0 \leq t \leq t_{m}$, so we have:

$$
\begin{aligned}
\|X(p)\| & =\left\|X(p)-X\left(p_{*}\right)\right\| \\
& \leq L\left\|p-p_{*}\right\| \\
& =L\left\|\phi_{t}^{j}\left(p_{m}\right)-\phi_{t}^{j}\left(p_{*}\right)\right\| \\
& \leq C\left\|p_{m}-p_{*}\right\|,
\end{aligned}
$$

where $L$ is the Lipschitz constant of $X$ and $C=L \max \left\{\left\|T_{q} \phi_{t}^{j}\right\|: 0 \leq t \leq\right.$ $\left.T_{\infty}, q \in K\right\}<\infty$. From this inequality we get that

$$
\|X\|_{A_{m}}:=\max _{p \in A_{m}}\|X(p)\| \leq C\left\|p_{m}-p_{*}\right\|
$$

for all $m \geq 0$.

Next observe that

$$
\left|A_{m}\right| \leq t_{m}\|X\|_{A_{m}},
$$

so $\left|A_{m}\right| /\|X\|_{A_{m}} \rightarrow 0$, as $m \rightarrow \infty$. However, by the non-zero angle requirement in (A2), there exists a constant $a>0$ such that $\left|A_{m}\right| \geq a\left\|p_{m}-p_{*}\right\|$, for all $m \geq 0$. Thus:

$$
\frac{\left|A_{m}\right|}{\|X\|_{A_{m}}} \geq \frac{a}{C}>0
$$

a contradiction. Therefore, $X\left(p_{*}\right) \neq 0$.

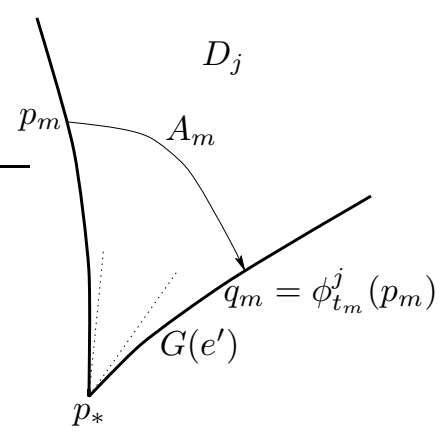

Figure 14: Proof of Proposition 4.4. 
The following example indicates that the non-zero angle assumption in (A2) was necessary in Proposition 4.4.

Example 4.5 (equilibrium + cusp = Zeno). Consider the following one-domain hybrid system (see Fig. 15):

$$
\begin{gathered}
D=\left\{(x, y) \in \mathbb{R}^{2}: y \geq 0, \quad-f(y) \leq x \leq f(y)\right\} \\
G=\{(-f(y), y): y \geq 0\}, \quad R(-f(y), y)=(f(c y), c y), \\
X(x, y)=(-x-y, x-y)^{T} .
\end{gathered}
$$

Here $0<c<1$ and $f(y)=y^{2}$ (in fact, any smooth $f$ such that $0 \leq f(y) \leq y^{2}$ would do). Since $f^{\prime}(0)=0, D$ has a cusp at $\mathbf{0}$.

The vector field $X$ has a spiral sink at the origin, and the time $t$ map of its flow is the composition of the counterclockwise rotation by $t$ (in radians) and contraction by $e^{-t}$. Let $p_{0}$ be an arbitrary nonzero point on the right part $S$ of the boundary of $D$ and let $\chi$ the execution starting from $p_{0}$. Let $\left(p_{m}\right)$ be the sequence of intersections of $\chi$ with $S$; let $p_{m}=\left(f\left(y_{m}\right), y_{m}\right)$. Let $t_{m}$ be the time it takes for the positive $X$-orbit of $p_{m}$ to reach $G$. Then $\left\|p_{m+1}\right\|=c e^{-t_{m}}\left\|p_{m}\right\|$, so

$$
\left\|p_{m}\right\|=c^{m} \exp \left(-\sum_{i=0}^{m-1} t_{i}\right)\left\|p_{0}\right\|, \quad \text { and } \quad y_{m} \leq c^{m} y_{0}
$$

Let $\theta_{m}$ be the angle between the line $\mathbf{0} p_{m}$ and the positive $y$-axis and $\eta_{m}$ the angle between the positive $y$-axis and the line $\mathbf{0} p_{m}^{\prime}$, where $p_{m}^{\prime}$ is the intersection of the positive $X$-orbit of $p_{m}$ and $G$. Then

$$
t_{m}=\theta_{m}+\eta_{m} \leq 2 \theta_{m}=2 \arctan \frac{f\left(y_{m}\right)}{y_{m}} \leq 2 y_{m} \leq 2 c^{m} y_{0}
$$

Therefore, $\sum t_{m}$ converges and $\mathbf{0}$ is a Zeno state despite the fact that it is an equilibrium for $X$. This shows the importance of geometry of domains and assumption (A2).

Before we proceed, we need to remind the reader of the following flow box theorem for smooth flows [31]. Namely, assume that $X$ is a smooth vector field on an open set $U \subset \mathbb{R}^{n}, p \in U$ and $X(p) \neq 0$. Then there exists a neighborhood $V$ of $p$ in $U$ (called a flow box for $X$ at $p$ ) and a smooth coordinate system on $V$ relative to which $X$ equals the vector field $\partial / \partial x_{1}$, i.e., the flow of $X$ looks like

$$
\phi_{t}\left(x_{1}, \ldots, x_{n}\right)=\left(x_{1}+t, x_{2}, \ldots, x_{n}\right) .
$$

This means that in a neighborhood of any of its nonsingular points, the flow of a smooth vector field has a particularly simple form. 


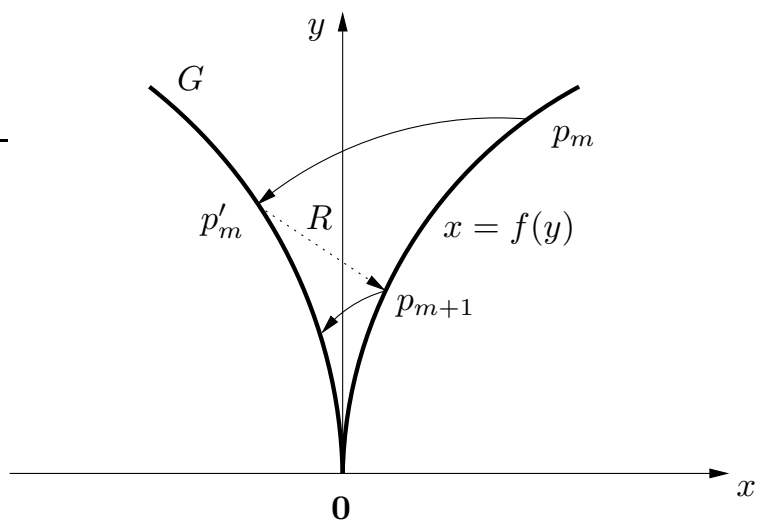

Figure 15: Zeno state at a cusp which is an equilibrium.

Theorem 4.6. Suppose that $M_{\mathbf{H}}$ has a smooth structure which makes the projection $\pi$ smooth and assume the hybrid flow $\Psi^{\mathbf{H}}$ is smooth with respect to that structure. Then $\mathbf{H}$ admits no Zeno executions or equivalently, there are no Zeno states in $M_{\mathbf{H}}$.

Proof. Fix a smooth structure on $M_{\mathbf{H}}$ which turns $\pi$ into a smooth map, and assume that there exists a Zeno state $z \in M_{\mathbf{H}}$ for some point $x_{0} \in M_{\mathbf{H}}$. Since the hybrid flow $\Psi^{\mathbf{H}}$ is assumed to be smooth, it is generated by a smooth vector field $Y$ on $M_{\mathbf{H}}$. The projection $\pi$ is smooth, so $Y(x)=T \pi\left(X_{i}(p)\right)$, for any point $p$ such that $\pi(p)=x$, where $X_{i}$ is the vector field on the domain containing $x$. By Proposition 4.4, $X_{i}(p) \neq 0$, for all $p \in \pi^{-1}(z)$ with $p \in D_{i}$. Observe that on $\bar{G} \cup \bar{R}, \pi$ is not one-to-one. However, $\pi$ does have the property that $T \pi(v) \neq 0$, for every vector nonzero $v$ tangent to $D$. Therefore, $Y(z) \neq 0$. By the Flow Box Theorem applied to a neighborhood of $z, \Psi^{\mathbf{H}}$ is the trivial horizontal flow (equivalent to the flow of the vector field $\partial / \partial x_{1}$ on $\left.\mathbb{R}^{n}\right)$, which implies that $z=\Psi_{\tau_{\infty}(x)}^{\mathbf{H}}(x)$. But then $t \mapsto \Psi_{t}^{\mathbf{H}}(x)$ is clearly defined beyond the Zeno time $\tau_{\infty}(x)$, which is contrary to our assumption.

In general it may not be easy to check whether, given $\mathbf{H}$, the hybrifold $M_{\mathbf{H}}$ is smooth. Even if it were, non-smoothness of the hybrid flow itself may cause Zeno to occur (cf., BB(2)). However, assuming that smoothness of the hybrifold can be verified, the following result provides an easily verifiable criterion for smoothness of $\Psi^{\mathbf{H}}$.

Theorem 4.7. Suppose that $M_{\mathbf{H}}$ is smooth in the same sense as in the previous theorem, and for every $e=(i, j) \in E$, there exists a smooth extension $\tilde{R}_{e}$ of $R_{e}$ such that $X_{i}$ and $X_{j}$ are $\tilde{R}_{e}$-related on $\overline{G(e)}$. That is, for every $p \in \overline{G(e)}$,

$$
T \tilde{R}_{e}\left(X_{i}(p)\right)=X_{j}\left(\tilde{R}_{e}(p)\right) .
$$


Then the hybrid flow is smooth.

Proof. Define a vector field $Y$ on $M_{\mathbf{H}}$ as follows. If $x \in M_{\mathbf{H}}$, then $x=\pi(p)$ for some $p \in D_{i}$. Set

$$
Y(x)=T \pi\left(X_{i}(p)\right)
$$

We will show that $Y$ is well defined.

If $p$ is not in $\bar{G} \cup \bar{R}$ (i.e., $p / \sim$ is a single point), then there is no ambiguity in the definition of $Y(x)$.

If $p \in \bar{G} \cup \bar{R}$, then the ambiguity arises because $p$ is identified with some $p^{\prime}$ via an extended reset map. Assume that $p \in \overline{G(e)}$ for some $e \in E$ and let $p^{\prime}=\tilde{R}_{e}(p)$. Since $\pi \circ \tilde{R}_{e}=\pi$, (4) and the chain rule yield

$$
\begin{aligned}
T \pi\left(X_{j}\left(p^{\prime}\right)\right) & =T \pi T \tilde{R}_{e}\left(X_{i}(p)\right) \\
& =T\left(\pi \circ \tilde{R}_{e}\right)\left(X_{i}(p)\right) \\
& =T \pi\left(X_{i}(p)\right) .
\end{aligned}
$$

Therefore, $Y$ is well defined.

Next we show that $Y$ is smooth. Let $X$ be the vector field on the total domain $D$ which coincides with $X_{i}$ on $D_{i}$, for all $i \in Q$. That is, $X$ is the union of $X_{i}$ 's. Since $D_{i}$ 's are mutually disjoint and each $X_{i}$ is smooth, $X$ is smooth. By definition of $Y$, the vector fields $X$ and $Y$ are $\pi$-related. Therefore, since $X$ and $\pi$ are smooth, so is $Y$.

Smoothness of $\Psi^{\mathbf{H}}$ now follows directly from the fact that $Y$ generates it, i.e.,

$$
\left.\frac{d}{d t}\right|_{0} \Psi_{t}^{\mathbf{H}}(x)=Y(x)
$$

for all $x \in M_{\mathbf{H}}$.

Example 4.8. Consider $B B(2)$. Here we have

$$
X_{1}\left(1, x_{1}, x_{2}\right)=\left(x_{2},-g\right)^{T}=X_{2}\left(2, x_{1}, x_{2}\right), \quad \tilde{R}_{(i, j)}\left(i, x_{1}, x_{2}\right)=\left(j, x_{1},-c x_{2}\right),
$$

where $(i, j)=(1,2)$ or $(2,1)$. Therefore,

$$
T \tilde{R}_{(1,2)}\left(X_{1}\right)=\left(x_{2}, c g\right)^{T} \neq X_{2},
$$

so the hybrid flow for $B B(2)$ is not smooth, as expected, since $B B(2)$ is Zeno.

Example 4.9. It is not difficult to check that in the case of $T^{2}(\alpha),(4)$ is satisfied for every $\alpha$. Thus $T^{2}(\alpha)$ does not accept Zeno executions, as was already shown above.

Corollary 4.10. If $M_{\mathbf{H}}$ is smooth in the sense of Theorem 4.6 and $\mathbf{H}$ satisfies condition (4), then $\mathbf{H}$ accepts no Zeno executions.

A Zeno state is an equilibrium of a sort. So we can define its stable and unstable sets in a standard way. 
Definition 4.11. Let $z$ be an isolated Zeno state and let $U \subset M_{\mathbf{H}}$ be its neighborhood. The set

$$
Z_{U}^{s}(z)=\{x \in U: \omega(x)=\{z\} \text { and the forward execution of } x \text { stays in } U\}
$$

is called the local stable set of $z$.

The local unstable set $Z_{U}^{u}(z)$ of $z$ is defined as the stable set of $z$ relative to the reverse hybrid system. We also define the global stable set of $z$ by

$$
Z^{s}(z)=\left\{\Psi_{t}^{\mathbf{H}}(x): x \in Z_{U}^{s}(z), t \leq 0, t \in J(x)\right\},
$$

where $U$ is any neighborhood of $z$ in $M_{\mathbf{H}}$. Thus $Z^{s}(z)$ is the union of all backward iterates of the local stable set of $z$. The global unstable set of $z$ is defined as the union of all forward iterates of the local unstable set of $z$. For instance, if $\mathbf{H}=W T, B B$ or $B B(m)$, then $Z^{s}(\mathbf{0})$ is a neighborhood of $\mathbf{0}$ (in fact, equals $M_{\mathbf{H}}$ ). We will see in the next section that this is not a coincidence. Namely, in dimension two, the global stable set of an isolated Zeno state is a neighborhood of the Zeno state. In higher dimensions, this is not the case, as the following simple example illustrates.

Example 4.12. The idea is to take the water tank example and add another direction along which the system is unstable. The system decouples into its $\left(x_{1}, x_{2}\right)$ - and $x_{3}$-part. More precisely, we have (cf., Example 2.2) $n=3$, $k=2, E=\{(1,2),(2,1)\}, D_{i}=\{i\} \times C \times \mathbb{R}$, where $C=[0, \infty) \times[0, \infty)$,

$$
X_{1}\left(1, x_{1}, x_{2}, x_{3}\right)=\left(w-v_{1},-v_{2}, 0\right)^{T}, \quad X_{2}\left(2, x_{1}, x_{2}, x_{3}\right)=\left(-v_{1}, w-v_{2}, 0\right)^{T},
$$

where $\max \left(v_{1}, v_{2}\right)<w<v_{1}+v_{2}$,

$$
\begin{aligned}
& G(1,2)=\left\{\left(1, x_{1}, x_{2}, x_{3}\right) \in D_{1}: x_{2}=0\right\}, \\
& G(2,1)=\left\{\left(2, x_{1}, x_{2}, x_{3}\right) \in D_{2}: x_{1}=0\right\},
\end{aligned}
$$

and

$$
R_{(1,2)}\left(1, x_{1}, 0, x_{3}\right)=\left(2, x_{1}, 0,2 x_{3}\right), \quad R_{(2,1)}\left(2,0, x_{2}, x_{3}\right)=\left(1,0, x_{2}, 2 x_{3}\right) .
$$

The hybrifold of the described system is homeomorphic to $\mathbb{R}^{3}$. The only Zeno state is $\mathbf{0}$ and its stable and unstable set are the $x_{1} x_{2}$-plane and the $x_{3}$-axis respectively. Executions starting from points with $x_{3}=0$ spiral around the $x_{3}$-axis and converge to $\mathbf{0}$ in finite time. Executions starting from points with $x_{3} \neq 0$ spiral around the $x_{3}$-axis, but escape to infinity in finite time.

Open question: When is a Zeno state hyperbolic? That is, under which conditions are the global stable and unstable set of a Zeno state smooth, transversely intersecting manifolds of complementary dimension? Note that this means there is no "center manifold" passing through the Zeno state. 


\section{Equivalence of hybrid systems}

In this section we discuss the following question: when are two hybrid systems qualitatively the same? For that purpose we borrow the notion of equivalence from the classical theory of dynamical systems [31]. Roughly speaking, two dynamical systems are (topologically) equivalent if their phase portraits look qualitatively (or topologically) the same. Similarly, two hybrid systems are equivalent if their hybrid flows are equivalent. We now make this more precise.

Definition 5.1. Two hybrid systems $\mathbf{H}_{\mathbf{1}}$ and $\mathbf{H}_{\mathbf{2}}$ are said to be topologically equivalent, denoted by $\mathbf{H}_{\mathbf{1}} \approx \mathbf{H}_{\mathbf{2}}$, if there exists a homeomorphism $h: M_{\mathbf{H}_{\mathbf{1}}} \rightarrow M_{\mathbf{H}_{\mathbf{2}}}$ which sends orbits of $\Psi^{\mathbf{H}_{\mathbf{1}}}$ to orbits of $\Psi^{\mathbf{H}_{\mathbf{2}}}$, preserving their orientation.

If $M_{\mathbf{H}_{1}}$ and $M_{\mathbf{H}_{\mathbf{2}}}$ happen to be smooth manifolds of class $C^{r}(r \geq 1)$ and $h$ is a $C^{r}$ diffeomorphism, then $\mathbf{H}_{\mathbf{1}}$ and $\mathbf{H}_{\mathbf{2}}$ are said to be $C^{r}$-equivalent.

As usual, by the orbit of a point $x$ under a (local) flow $\left\{\phi_{t}\right\}$ we mean the set of points $\phi_{t}(x)$ for all $t$ for which $\phi_{t}(x)$ is defined. We usually think of $h$ as a change of coordinates; so two hybrid systems are topologically equivalent if their hybrid flows are the same up to a continuous coordinate change. Note that equivalence does not necessarily preserve the time parameter $t$. If it does, it is called conjugacy.

Example 5.2. $W T$ is topologically equivalent to $B B$. This can be seen in the following way. Assume $M_{W T}$ is embedded in $\mathbb{R}^{3}$ in such a way that its "origin" coincides with the point $(0,0,0)$ and $M_{W T}$ lies entirely in the upper half space $\mathbb{R}_{+}^{3}$ and each vertical line intersects it exactly once. Let $P$ be the plane $x_{3}=0$ and let $h: M_{W T} \rightarrow P$ be the orthogonal projection. Then $h$ is a homeomorphism which sends orbits of $\Psi^{W T}$ to the orbits of the flow $\Phi$ in Fig. 16 .

By smoothing $\Phi$ along the $y$-axis, we obtain that it is topologically equivalent to a (smooth) spiral sink at the origin, e.g., the flow of the linear vector field $X(x, y)=(-x-y, x-y)^{T}$.

Similarly, we can show that also $\Psi^{B B}$ is topologically equivalent to the flow of the same $X$. Since topological equivalence is transitive, we obtain that $W T \approx B B$, as claimed. We will see later that in dimension two this picture is typical.

Example 5.3. $T^{2}(1)$ is not equivalent to $T^{2}(\sqrt{2})$. Even though the hybrifold for both hybrid systems is the same (the 2-torus), every orbit of $T^{2}(1)$ is closed, while every orbit of $T^{2}(\sqrt{2})$ is dense in $T^{2}$. Since equivalence always sends closed orbits to closed orbits, the statement above follows immediately.

Ideally, one would like to be able to classify all hybrid systems up to topological equivalence. Unfortunately, this attempt fails even for smooth dynamical systems on compact boundaryless manifolds of dimension greater 


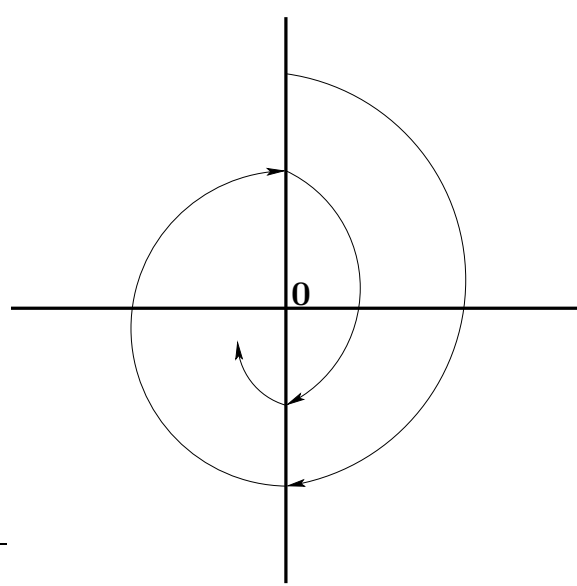

Figure 16: Flow in $\mathbb{R}^{2}$ topologically equivalent to $\Psi^{W T}$.

than two, as can be seen in the standard dynamics literature (for instance, [31]). However, it turns out that it is possible to obtain a complete picture of the local behavior of 2-dimensional hybrid flows near an isolated Zeno state. For completeness, we include an easy observation for 1-dimensional hybrid flows.

Theorem 5.4. Assume $\mathbf{H}$ is a regular hybrid system.

(a) If $\operatorname{dim} \mathbf{H}=1$, then $\mathbf{H}$ has no Zeno states.

(b) Suppose $\operatorname{dim} \mathbf{H}=2, \mathbf{H}$ is without branching, and $z \in M_{\mathbf{H}}$ is an isolated Zeno state. Then there is a neighborhood $U$ of $z$ in $M_{\mathbf{H}}$ and a neighborhood $V$ of $\mathbf{0}$ in $M_{W T}$ such that $\left.\Psi^{\mathbf{H}}\right|_{U}$ is topologically equivalent to $\left.\Psi^{W T}\right|_{V}$.

Proof. (a) Topologically, $M_{\mathbf{H}}$ must be either the circle or an interval (bounded or unbounded, open, closed or half-closed). Suppose $M_{\mathbf{H}}$ contains a Zeno state for some $x \in M_{\mathbf{H}}$. Then $\chi(x)$ must visit at least one domain infinitely often, so by construction, $M_{\mathbf{H}}$ must be a circle. Therefore, $\chi(x)$ must go around this circle and hence must be the whole $M_{\mathbf{H}}$. This implies that its execution time must be infinite, which is a contradiction.

(b) Let $z$ be the Zeno state for some $x_{0} \in M_{\mathbf{H}}$. Then $\tau_{\infty}\left(x_{0}\right)>0$. Denote by $\chi_{0}$ the execution starting from $x_{0}$. Assume

$$
\pi^{-1}(z)=\left\{z_{1}, \ldots, z_{m}\right\}
$$

and $z_{j} \in D_{j}$ (if this is not the case, reorder the domains). Let $\tilde{\chi}_{0}=\pi^{-1}\left(\chi_{0}\right)$ and assume $\tilde{\chi}_{0}$ visits the domains $D_{1}, \ldots, D_{l}$ infinitely often, for some $1 \leq$ 
$l \leq m$ (if necessary, reorder the domains). Then $\tilde{\chi}_{0}$ must accumulate at $z_{1}, \ldots, z_{l}$. Suppose that starting from $D_{1}$, it goes through $D_{2}, \ldots, D_{l}$ in that order. This means that $e_{j}=(j, j+1)$, for $j=1, \ldots, l-1$, are all allowed transitions, i.e., $e_{j} \in E$. Denote

$$
A_{j}=\operatorname{im} R_{e_{j-1}}, \quad B_{j}=G\left(e_{j}\right)
$$

It is easy to see that $z_{j} \in \overline{A_{j}} \cap \overline{B_{j}}(1 \leq j \leq l-1)$. At this point, after passing through $D_{1}, \ldots, D_{l-1}$, and completing its evolution through $D_{l}, \tilde{\chi}_{0}$ has to switch to one of the domains $D_{1}, \ldots, D_{l}$. We show that it must switch to $D_{1}$. To see this, observe that at $z_{j}$ (as at any other boundary point of any guard or image of a reset), $A_{j}, B_{j}, \partial A_{j}$, and $\partial B_{j}$ all have to look like manifolds of appropriate dimensions. Therefore, since $\operatorname{dim} D_{j}=2$, there is no guard in $D_{j}$, other than $B_{j}$, containing $z_{j}$ in its boundary, and there is no reset, other than $R_{(j-1, j)}$ whose image contains $z_{j}$ in its boundary. Assume now that from $D_{l-1}, \tilde{\chi}_{0}$ switches to $D_{r}$, for some $r \neq 1$. Then, again since $\tilde{\chi}_{0}$ accumulates at $z_{1}, \ldots, z_{l}$, and by the previous observation about the structure of $A_{j}$ 's and $B_{j}$, after completing its evolution through $D_{r}$, it will be forced to switch to $D_{r+1}$, etc., therefore avoiding $D_{1}$ altogether in the future, which is a contradiction.

Therefore, we can, without loss, assume that $\tilde{\chi}_{0}$ visits $D_{1}, D_{2}, \ldots, D_{l}, D_{1}, D_{2}, \ldots$, respectively.

Next, the basic observation is: since $X_{j}\left(z_{j}\right) \neq 0$, for every $j$, no trajectory of $X_{j}$ can reach $z_{j}$ in finite time. Otherwise, it would prevent $\chi_{0}$ from making infinitely many turns around $z$. Details follow.

1. We claim that for each $j$ there exists $V_{j}$, a neighborhood of $z_{j}$ in $D_{j}$, such that every execution starting in $A_{j} \cap V_{j}-\left\{z_{j}\right\}$ reaches $B_{j} \cap V_{j}-\left\{z_{j}\right\}$.

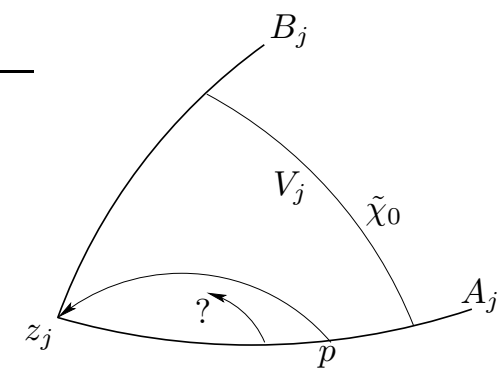

Figure 17: The execution starting at $p$ cannot reach $z_{j}$.

To prove this, let $V_{j}$ be the region in $D_{j}$ bounded by $A_{j}, B_{j}$ and a single arc of $\tilde{\chi}_{0}$, and let $p \in A_{j} \cap V_{j}-\left\{z_{j}\right\}$ be an arbitrary point; see Fig. 17. The execution $\chi(p)$ cannot intersect $\tilde{\chi}_{0}$, so it must reach $\overline{B_{j}} \cap V_{j}$. If it passes through $z_{j}$ before it reaches $B_{j} \cap V_{j}-\left\{z_{j}\right\}$, then every execution starting from a point in $\overline{A_{j}}$ between $p$ and $z_{j}$ must pass through $z_{j}$. This is impossible 
since, according to Proposition $4.4, z_{j}$ is not an equilibrium of $X_{j}$ (here we also used the fact that $X_{j}$ points inside $D_{j}$ along $A_{j}$ ). Therefore, $\chi(p)$ reaches $B_{j} \cap V_{j}-\left\{z_{j}\right\}$.

Let $V=\bigcup V_{j}$ and $U=\pi(V)$.

2. We now investigate the only two possibilities:

Case $1: \partial D_{j}$ is smooth at $z_{j}$. Then it follows immediately from (A6) and Proposition 4.4 that $X_{j}$ is tangent to $\partial D_{j}$ at $z_{j}$. Therefore, by $\mathbf{1}$., in a smooth flow box around $z_{j}$, the local picture is as in part (A) of Fig. 18. (Recall that $X_{j}$ extends smoothly to a neighborhood of $D_{j}$.)

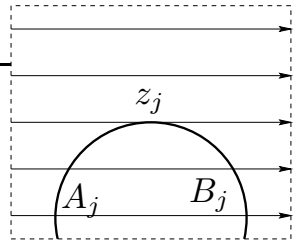

(A)

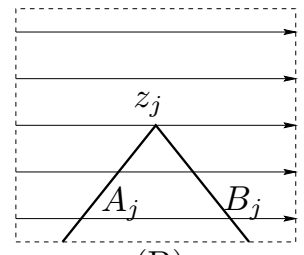

(B)

Figure 18: Local picture around $z_{j}$.

Case $2: \partial D_{j}$ is not smooth at $z_{j}$. Because of $\mathbf{1}$., it is not difficult to see that the local picture around $z_{j}$ looks like part (B) of Fig. 18.

3. In fact, in both cases, up to a continuous change of coordinates, the local picture around $z_{j}$ looks like part (B) of Fig. 18, but with $A_{j}$ and $B_{j}$ being straight line segments. To construct a topological equivalence between $\Psi^{\mathbf{H}}$ near $z_{j}$ and $\Psi^{W T}$ near $\mathbf{0}$, subdivide $D_{1}$ of $W T$ into $l-1$ subdomains $D_{1}^{\prime}, \ldots, D_{l-1}^{\prime}$ by $l-2$ rays from the origin. Let $D_{l}^{\prime}=D_{2}$. Define a hybrid system $W T_{l}$ by: the domains are $D_{1}^{\prime}, \ldots, D_{l}^{\prime}$, the vector fields $X_{j}^{\prime}$ are the restrictions of the vector fields of $W T$ to the corresponding new domains, and the resets are identity maps.

It is easily seen that $X_{j}$ on $V_{j}$ is topologically equivalent to $X_{j}^{\prime}$ on $D_{j}^{\prime}$. Call the conjugating homeomorphism $h_{j}$. Glue the $h_{j}$ 's together to obtain a homeomorphism $h$ between $\Psi^{\mathbf{H}}$ on $U$ and $\Psi^{W T_{l}}$ on a neighborhood of 0. Since $\Psi^{W T_{l}}$ is clearly equivalent to $\Psi^{W T}$, the proof of the theorem is complete.

\section{Concluding remarks}

We study a class of hybrid systems which can be identified with piecewise smooth continuous-time dynamical systems on piecewise smooth manifolds. This is the class of "regular hybrid systems with no branching". In our framework, which includes many known examples and makes the hybrid systems 
under consideration manageable for analysis, we investigate the Zeno phenomenon, show that it is due to the lack of smoothness, and classify it locally in dimension two.

This article is an initial step towards a global geometric theory of hybrid systems. Some work in the same direction can be found in $[12,21,34,35]$, where we studied structural stability, stability of equilibria, and stability of closed orbits of hybrid systems. We are interested in global geometric and topological properties of a larger class of hybrid systems, including nondeterministic ones, examples of "chaotic" behavior in dimension two (which would further distinguish hybrid systems from smooth 2-dimensional flows) and different ways of studying stability of invariant sets, including Zeno ones. We postpone the discussion of these and other question to our forthcoming articles.

\section{References}

[1] C. Altafini, A. Speranzon, and K. H. Johansson. Hybrid control of a truck and trailer vehicle. In C. J. Tomlin and M. R. Greenstreet, editors, Hybrid Systems: Computation and Control, volume 2289 of Lecture Notes in Computer Science. Springer-Verlag, New York, 2002.

[2] R. Alur, C. Courcoubetis, and D. Dill. Model checking for real-time systems. In Proc. 5th Ann. IEEE Symp. on Logic in Computer Science, pages 414-425, Philadelphia, PA, 1990.

[3] R. Alur, C. Courcoubetis, N. Halbawachs, T. A. Henzinger, P. H. Ho, X. Nicollin, A. Olivero, J. Sifakis, and S. Yovine. The algorithmic analysis of hybrid systems. Theoretical Computer Science, 138:3-34, 1995.

[4] R. Alur and T.A. Henzinger. Modularity for timed and hybrid systems. In Proceedings of the Eighth International Conference on Concurrency Theory (CONCUR 1997), number 1243 in LNCS, pages 74-88. Springer Verlag, 1997.

[5] A. Balluchi, L. Benvenutti, M. Di Benedetto, C. Pinello, and A. SangiovanniVincentelli. Automotive engine control and hybrid systems: Challenges and opportunities. Proceedings of the IEEE, 7:888-912, July 2000.

[6] A. Balluchi, P. Soueres, and A. Bicchi. Hybrid feedback control for path tracking by a bounded-curvature vehicle. In M. di Benedetto and A. L. Sangiovanni-Vincentelli, editors, Hybrid Systems: Computation and Control, volume 2034 of Lecture Notes in Computer Science, pages 133-146. Springer-Verlag, New York, 2001.

[7] A. Bemporad and M. Morari. Control of systems integrating logic dynamics and constraints. Automatica, 35(3):407-427, March 1999.

[8] A. Bensoussan and J.L. Menaldi. Hybrid control and dynamic programming. Dynamics of Continuous, Discrete and Impulsive Systems, (3):395-442, 1997.

[9] M. S. Branicky. Multiple Lyapunov functions and other analysis tools for switched and hybrid systems. IEEE Transactions on Automatic Control, 43(4):475-482, 1998.

[10] M. S. Branicky, V. S. Borkar, and S. K. Mitter. A unified framework for hybrid control: Model and optimal control theory. IEEE Transactions on Automatic Control, 43(1):31-45, 1998.

[11] R. W. Brockett. Hybrid models for motion control systems. In H. Trentelman and J. Willems, editors, Essays in Control: Perspectives in the Theory and Its Applications, pages 29-53. Birkhäuser, Boston, 1993. 
[12] M. Broucke, C. Pugh, and S. N. Simić. Structural stability of piecewise smooth systems. Computational and Applied Mathematics, 20(1-2):51-89, 2001.

[13] S. Engell, S. Kowalewski, C. Schultz, and O. Strusberg. Continuous-discrete interactions in chemical process plants. Proceedings of the IEEE, 7:1050-1068, July 2000.

[14] A. F. Filippov. Differential equations with discontinuous righthand sides. Mathematics and its applications (Soviet series). Kluwer, 1988.

[15] M. Heemels. Linear Complementarity Systems: a Study in Hybrid Dynamics. PhD thesis, Technische Universiteit Eindhoven, 1999.

[16] M. Heemels, H. Schumacher, and S. Weiland. Well-posedness of linear complementarity systems. In Proc. 38th IEEE Conference on Decision and Control, Phoenix, AZ, 1999.

[17] T. Henzinger, P. Kopke, A. Puri, and P. Varaiya. What's decidable about hybrid automata. Journal of Computer and System Sciences, 57:94-124, 1998.

[18] M. Heymann, F. Lin, and G. Meyer. Synthesis and viability of minimally interventive legal controllers for hybrid systems. Discrete Event Dynamic Systems: Theory and Applications, 8(2):105-135, 1998.

[19] J-I. Imura and A.J. van der Schaft. Well-posedness of a class of piecewise linear systems with no jumps. In Frits W. Vaandrager and Jan H. van Schuppen, editors, Hybrid Systems: Computation and Control, number 1569 in LNCS, pages 123-136. Springer Verlag, 1999.

[20] K. H. Johansson, M. Egerstedt, J. Lygeros, and S. Sastry. On the regularization of Zeno hybrid automata. Systems and Control Letters, 38:141-150, 1999.

[21] K. H. Johansson, J. Lygeros, S.N. Simić, J. Zhang, and S. Sastry. Dynamical properties of hybrid automata. IEEE Transactions on Automatic Control, 48(1):2-17, 2003.

[22] M. Johansson and A. Rantzer. Computation of piecewise quadratic lyapunov functions for hybrid systems. IEEE Transactions on Automatic Control, 43(4):555-559, 1998.

[23] G. Lafferriere, G. J. Pappas, and Shankar Sastry. O-minimal hybrid systems. Mathematics of Control, Signals, and Systems, 13(1):1-21, March 2000.

[24] M. Lemmon. On the existence of solutions to controlled hybrid automata. In N. Lynch and B. H. Krogh, editors, Hybrid Systems: Computation and Control, number 1790 in LNCS, pages 229-242. Springer Verlag, New York, 2000.

[25] J. Lygeros, D. N. Godbole, and S. Sastry. A verified hybrid controller for automated vehicles. IEEE Transactions on Automatic Control, 43(4):522-539, 1998.

[26] J. Lygeros, K. H. Johansson, S. Sastry, and M. Egerstedt. On the existence of executions of hybrid automata. In IEEE Conference on Decision and Control, pages 2249-2254, Phoenix, Arizona, U.S.A., December 7-10 1999.

[27] J. Lygeros, C. Tomlin, and S. Sastry. Controllers for reachability specifications for hybrid systems. Automatica, pages 349-370, March 1999.

[28] N. Lynch, R. Segala, F. Vaandrager, and H.B. Weinberg. Hybrid I/O automata. In Hybrid Systems III, number 1066 in LNCS, pages 496-510. Springer Verlag, 1996.

[29] O. Maler, A. Pnueli, and J. Sifakis. On the synthesis of discrete controllers for timed systems. In Theoretical Aspects of Computer Science, number 900 in LNCS, pages 229-242. Springer Verlag, 1995.

[30] J. R. Munkres. Topology; a first course. Englewood Cliffs, NJ, 1974.

[31] J. Palis Jr. and W. de Melo. Geometric Theory of Dynamical Systems. Springer Verlag, New York, 1982.

[32] D.L. Pepyne and C.G. Cassandras. Optimal control of hybrid systems in manufacturing. Proceedings of the IEEE, 7:1108-1123, July 2000. 
[33] S. N. Simić, K. H. Johansson, S. Sastry, and J. Lygeros. Towards a geometric theory of hybrid systems. In N. Lynch and B. H. Krogh, editors, Hybrid Systems: Computation and Control, number 1790 in LNCS, pages 421-436. Springer Verlag, 2000.

[34] S. N. Simić, K.H. Johansson, J. Lygeros, and S. Sastry. Structural stability of hybrid systems. In European Control Conference, pages 3858-3863, Porto, Portugal, September 4-7 2001 .

[35] S. N. Simić, K.H. Johansson, J. Lygeros, and S. Sastry. Hybrid limit cycles and hybrid Poincaré-Bendixson. In IFAC World Congress, Barcelona, Spain, July 22-26 2002.

[36] L. Tavernini. Differential automata and their simulators. Nonlinear Analysis, Theory, Methods and Applications, 11(6):665-683, 1987.

[37] C. Tomlin, G. Pappas, and S. Sastry. Conflict resolution for air traffic management: A study in multiagent hybrid systems. IEEE Transactions on Automatic Control, 43(4):509-521, 1998.

[38] A.J. van der Schaft and H. Schumacher. Complementarity modeling of hybrid systems. IEEE Transactions on Automatic Control, 43(4):483-490, 1998.

[39] P. Varaiya. Smart cars on smart roads: Problems of control. IEEE Transactions on Automatic Control, 38(2):195-207, 1993.

[40] H. Ye, A. Michel, and L. Hou. Stability theory for hybrid dynamical systems. IEEE Transactions on Automatic Control, 43(4):461-474, 1998.

[41] J. Zhang, K. H. Johansson, J. Lygeros, and S. Sastry. Dynamical systems revisited: Hybrid systems with zeno executions. In N. Lynch and B. H. Krogh, editors, Hybrid Systems: Computation and Control, number 1790 in LNCS, pages 451-464. Springer Verlag, 2000.

[42] J. Zhang, K. H. Johansson, J. Lygeros, and S. Sastry. Zeno hybrid systems. International Journal of Robust and Nonlinear Control, 11(5):435-451, 2001.

email:journal@monotone.uwaterloo.ca

http://monotone.uwaterloo.ca/ journal/ 